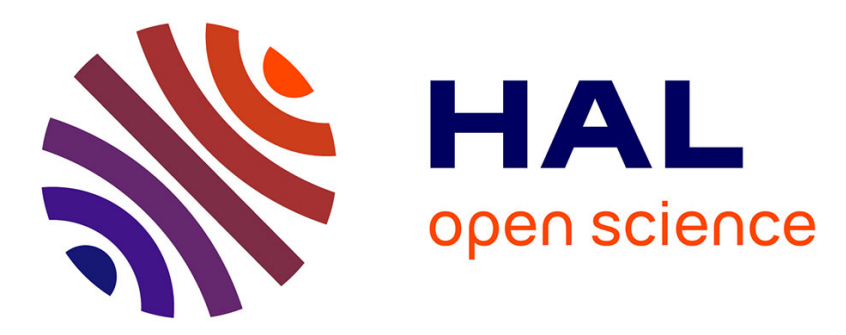

\title{
An overview of the numerical methods for tokamak plasma equilibrium computation implemented in the NICE code
}

\author{
Blaise Faugeras
}

\section{- To cite this version:}

Blaise Faugeras. An overview of the numerical methods for tokamak plasma equilibrium computation implemented in the NICE code. Fusion Engineering and Design, 2020, 160, pp.112020. 10.1016/j.fusengdes.2020.112020 . hal-02955053

\section{HAL Id: hal-02955053 \\ https://hal.science/hal-02955053}

Submitted on 1 Oct 2020

HAL is a multi-disciplinary open access archive for the deposit and dissemination of scientific research documents, whether they are published or not. The documents may come from teaching and research institutions in France or abroad, or from public or private research centers.
L'archive ouverte pluridisciplinaire HAL, est destinée au dépôt et à la diffusion de documents scientifiques de niveau recherche, publiés ou non, émanant des établissements d'enseignement et de recherche français ou étrangers, des laboratoires publics ou privés. 


\title{
An overview of the numerical methods for tokamak plasma equilibrium computation implemented in the NICE code
}

\author{
Blaise Faugeras ${ }^{\mathrm{a}}$ \\ ${ }^{a}$ Université Côte d'Azur, CNRS, INRIA Castor, LJAD, Parc Valrose, 06108 Nice \\ Cedex 2, France
}

\begin{abstract}
The code NICE (Newton direct and Inverse Computation for Equilibrium) enables to solve numerically several problems of plasma free-boundary equilibrium computations in a tokamak: plasma free-boundary only reconstruction and magnetic measurements interpolation, full free-boundary equilibrium reconstruction from magnetic measurements and possibly internal measurements (interferometry, classical linear approximation polarimetry or Stokes model polarimety, Motional Stark Effect and pressure), direct and inverse, static and quasi-static free-boundary equilibrium computations.

NICE unifies and upgrades 3 former codes VacTH [1], EQUINOX [2] and CEDRES ++ [3]. The strength of NICE is to gather in a single finite element framework different equilibrium computation modes. It makes intensive use of Newton method and Sequential Quadratic Programming method to solve non linear problems.

NICE is used routinely for WEST tokamak operation. It is also adapted to the IMAS (ITER Modelling and Analysis Suite) format which makes it usable on many different fusion tokamak reactors.

In this document we give a general overview of the numerical methods implemented in NICE as well as a number of computation examples.
\end{abstract}

Key words: tokamak, plasma equilibrium, equilibrium reconstruction, toroidal harmonics, finite element method, Newton method, inverse problem, PDE-constrained optimization

PACS: 02.03.Zz, 02.60.-x, 52.55.-s, 52.55.Fa, 52.65.-y

Email addresses: blaise.faugeras@univ-cotedazur.fr (Blaise Faugeras) 


\section{Introduction}

The aim of this paper is to provide a reference document on the different operation modes and numerical methods implemented in the code NICE. NICE stands for Newton direct and Inverse Computation for Equilibrium. Its development started in 2017 with the original goal of developing an equilibrium reconstruction code able to use polarimetry Stokes vector measurements. It demanded the computation of derivatives of certain quantities which did not easily fit in the equilibrium reconstruction code EQUINOX [2] developed by the author and others. This led to the development and implementation of the optimal control algorithm, presented in this document, with a first step presented in [4], which can naturally deal with the non-linear Stokes equations. Moreover it soon appeared appealing to unify in a single performant $\mathrm{C}++$ code the different functionalities from 3 older codes, VacTH [1], EQUINOX [2] and CEDRES++ [3] which share many common features. These goals are now achieved and on the way, improvements to the original methods and new functionalities were added.

Several problems of equilibrium computation can be addressed by NICE and are detailed in this document:

- plasma boundary only reconstruction from magnetic measurements (Section 3). The reference paper for this mode is [1]. In addition to the method proposed in this reference the use of an original regularization term which proved to be efficient is proposed in this document.

- full equilibrium reconstruction from magnetic measurements and possibly internal measurements (Section 4). NICE uses Sequential Quadratic Programming (SQP) algorithms to solve partial differential equation (PDE) constrained optimization problems and in particular the inverse problem of equilibrium reconstruction. The default algorithm used in NICE is a quasi SQP with reduced Hessian algorithm (which we denote QSQP in this document). Its implementation demands the computation of derivatives of quantities with respect to the state and control variables and enables to use polarimetry Stokes vector measurements [4, 5]. The code also incorporates the original optimization algorithm from EQUINOX [2] which appears to be an approximation of the default QSQP algorithm used in NICE. A sensitivity method for error 
bar computations on every reconstructed equilibrium quantities is also implemented and detailed in Section 4.3.2.

- direct and inverse, static and quasi-static free-boundary equilibrium computations (Section 5). The reference papers for these implemented modes are [3] and [6]. The term direct refers to the resolution of the equilibrium equation whereas the term inverse refers to the inverse problem consisting in finding the currents in the poloidal field (PF) coils which give a desired plasma shape in the static case or the voltages in the suppliers which give a desired evolution of the plasma shape in the quasi-static case. The difference between static and quasi-static is explained in Section 2.1

The strengh of NICE is to gather in a single unified framework different functionalities or equilibrium computation modes. It makes intensive use of Newton method and SQP method to solve non linear problems.

NICE is used routinely for the WEST tokamak operation [7]. NICE is adapted to the IMAS (ITER Modelling and Analysis Suite [8, 9]) format which makes it usable on many different tokamaks [10]. Many of the numerical examples provided in this document are obtained using data in the IMAS format.

The document is organized as follows. Section 2 provides a brief description of the equilibrium equations used (Section 2.1), of their finite element discretization (Section 2.2) and of the iterative Newton and QSQP algorithm used (Section 2.3). This section enables to set matrix notations which are very close to what is used in the code itself. Section 3 describes the plasma boundary only reconstruction mode of NICE. This first mode does not rely on finite element discretization but uses a decomposition of the poloidal flux in toroidal harmonics. All other computation modes of NICE use finite elements. Section 4 presents the equilibrium reconstruction mode and Section 5 the direct and inverse equilibrium computation modes. For each mode numerical examples are provided.

\section{Tokamak free-boundary plasma equilibrium}

This section presents the general equations which are dealt with in NICE. The finite element discretization is also presented which enables us to set the notations for different matrix and vector operators which are used in the different modes of NICE. Then we present in a generic framework the QSQP 
algorithm which will then be applied to the inverse problems of the next sections.

\subsection{Modelization}

The equations which govern the equilibrium of a plasma. in the presence of a magnetic field in a tokamak are on the one hand Maxwell's equations satisfied in the whole of space (including the plasma):

$$
\nabla \cdot \mathbf{B}=0, \quad \nabla \times\left(\frac{\mathbf{B}}{\mu}\right)=\mathbf{j}
$$

and on the other hand the equilibrium equation for the plasma itself

$$
\nabla p=\mathbf{j} \times \mathbf{B},
$$

where $\mathbf{B}$ is the magnetic field, $\mu$ is the magnetic permeability, $p$ is the kinetic pressure and $\mathbf{j}$ is the current density.

These equations are sufficient for the static modelization of the plasma equilibrium. In this modelization the current densities in the PF coils are given. In case of quasi-static plasma evolution modelization these equations are augmented with Faraday's law and Ohm's law in the PF coils and passive structures. The current density in these structures is then computed as a function of the input voltages in the PF circuits and/or of the time derivative of the poloidal flux (defined below) as shown in Eqs. (11) and (12) below. We refer to standard text books (e.g. [11, 12, 13, 14, 15]) and to [2, 3] for details of the derivation and only state the needed equations in what follows.

Introducing a cylindrical coordinate system $\left(\boldsymbol{e}_{r}, \boldsymbol{e}_{\phi}, \boldsymbol{e}_{z}\right)(r=0$ is the major axis of the tokamak torus) and assuming axial symmetry equations (1) and (2) reduce to the following equation for the poloidal flux $\psi(r, z)$ in the poloidal plane $\Omega_{\infty}=(0, \infty) \times(-\infty, \infty)$ :

$$
-\Delta^{*} \psi=j_{\phi}
$$

where $j_{\phi}$ is the toroidal component of $\mathbf{j}$, and the second order elliptic differential operator $\Delta^{*}$ is defined by

$$
\Delta^{*} \psi:=\partial_{r}\left(\frac{1}{\mu(\psi) r} \partial_{r} \psi\right)+\partial_{z}\left(\frac{1}{\mu(\psi) r} \partial_{z} \psi\right):=\nabla \cdot\left(\frac{1}{\mu(\psi) r} \nabla \psi\right)
$$

Here $\nabla$ is the $2 \mathrm{D}$ operator in the $(r, z)$-plane and $\mu(\psi)$ is the magnetic permeability. It is equal to the constant permeability of vacuum $\mu_{0}$ everywhere 
except in the possibly existing iron parts of the tokamak (see Fig. 1) where it is a given function of $\psi, \mu(\psi)=\mu_{f}\left(|\nabla \psi|^{2} r^{-2}\right)$.

The magnetic field can be decomposed in poloidal and toroidal components

$$
\mathbf{B}=\mathbf{B}_{p}+\mathbf{B}_{\phi}, \quad \mathbf{B}_{p}=\frac{1}{r}\left[\nabla \psi \times \boldsymbol{e}_{\phi}\right], \quad \boldsymbol{B}_{\phi}=B_{\phi} \mathbf{e}_{\phi}=\frac{f}{r} \boldsymbol{e}_{\phi},
$$

where $f$ is the diamagnetic function. Equation (5) shows that the magnetic surfaces are generated by the rotation of the iso-flux lines around the axis of the torus.

The toroidal component of the current density. $j_{\phi}$ is zero everywhere outside the plasma domain and the poloidal field coils (and possibly the passive structures). The different sub-domains of the poloidal plane of a schematic tokamak (see Figure 1) as well as the corresponding expression for $j_{\phi}$ are described below:

$-\Omega_{\mathrm{f}}$ is the domain of ferromagnetic iron structures where the permeability $\mu$ is not constant.

$-\Omega_{\mathrm{L}}$ is the domain accessible to the plasma. Its boundary is the limiter $\Gamma_{L}$.

$-\Omega_{\mathrm{p}}$ is the plasma domain where equations $(2)$ and $(1)$ imply that $p$ and $f$ are constant on each magnetic surface i.e. $p=p(\psi)$ and $f=f(\psi)$. One then deduces the so-called Grad-Shafranov equilibrium equation in the plasma [16, 17, 18.

$$
-\Delta^{*} \psi=r p^{\prime}(\psi)+\frac{1}{\mu_{0} r}\left(f f^{\prime}\right)(\psi) .
$$

The right-hand side of (6) is the toroidal component $j_{\phi}$ of the plasma current density.

The plasma domain depends on $\psi$ and is unknown, $\Omega_{\mathrm{p}}=\Omega_{\mathrm{p}}(\psi)$. We have to deal with a free-boundary problem. This domain is defined by its boundary which is the outermost closed $\psi$ iso-contour contained within the limiter region $\Omega_{\mathrm{L}}$. The plasma can either be limited if this iso-contour is tangent to the limiter $\Gamma_{L}$ or defined by the presence of an X-point (as in Figure 1). In the latter case the plasma domain is bounded by the magnetic separatrix. Functions $p^{\prime}$ and $f f^{\prime}$ are zero outside $\Omega_{\mathrm{p}}$.

The current density is non-linear in $\psi$ due to the non-linear functions $p^{\prime}$ and $f f^{\prime}$ and the definition of the plasma domain $\Omega_{\mathrm{p}}(\psi)$. While $\Omega_{\mathrm{p}}(\psi)$ is fully determined for a given $\psi$, the two functions $p^{\prime}$ and $f f^{\prime}$ are not determined in this modelization. It is the goal of the inverse equilibrium reconstruction 


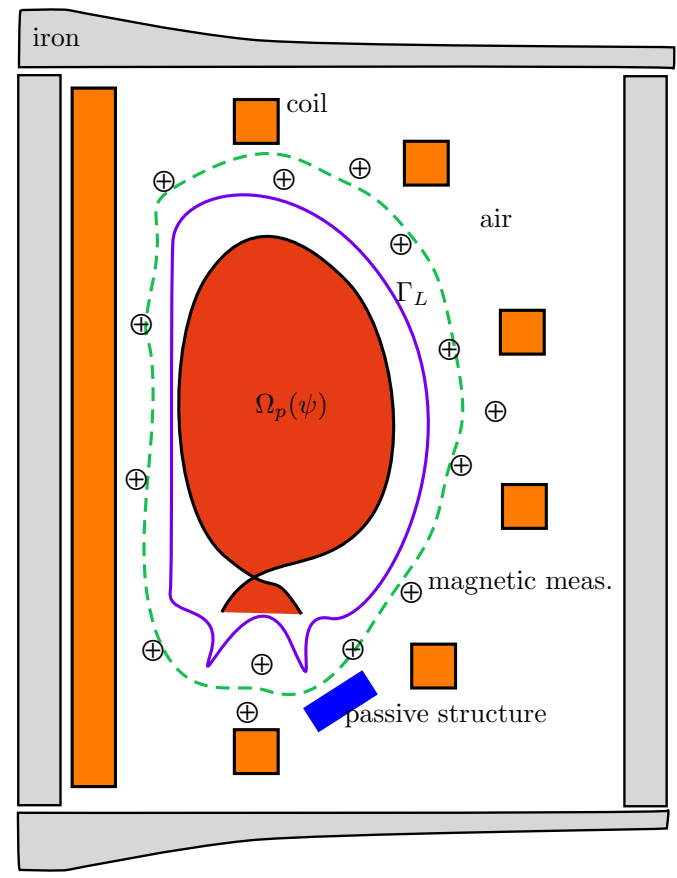

Figure 1: Schematic representation of the poloidal plane of a tokamak. Ferromagnetic iron structures $\left(\Omega_{\mathrm{f}}\right)$ are represented in gray, PF coils $\left(\Omega_{\mathrm{c}_{i}}\right)$ in orange and a passive structure $\left(\Omega_{\mathrm{ps}}\right)$ in blue. The limiter contour $\left(\Gamma_{L}\right.$, purple contour) inside which the plasma $\left(\Omega_{\mathrm{p}}\right)$ lies is also shown. Cross-circles represent magnetic probes and a fictitious measurement contour $\Gamma$ is drawn with a green dashed line. 
problem to determine them. For now let us consider that we are given two functions $\mathcal{A}(x)$ and $\mathcal{B}(x)$ defined on $[0,1]$ such that in the plasma domain $\Omega_{\mathrm{p}}(\psi)$

$$
j_{\phi}=\lambda\left(\frac{r}{r_{0}} \mathcal{A}\left(\psi_{\mathrm{N}}\right)+\frac{r_{0}}{r} \mathcal{B}\left(\psi_{\mathrm{N}}\right)\right) .
$$

Here $r_{0}$ is the characteristic major radius of the tokamak vacuum chamber and $\lambda$ is a scaling coefficient. The normalized poloidal flux $\psi_{\mathrm{N}}(r, z)$ is

$$
\psi_{\mathrm{N}}(r, z)=\frac{\psi(r, z)-\psi_{\mathrm{a}}(\psi)}{\psi_{\mathrm{b}}(\psi)-\psi_{\mathrm{a}}(\psi)}
$$

with $\psi_{\mathrm{a}}$ and $\psi_{\mathrm{b}}$ being the flux values at the magnetic axis and at the boundary of the plasma:

$$
\begin{aligned}
\psi_{\mathrm{a}}(\psi) & :=\psi\left(r_{\mathrm{a}}(\psi), z_{\mathrm{a}}(\psi)\right), \\
\psi_{\mathrm{b}}(\psi) & :=\psi\left(r_{\mathrm{b}}(\psi), z_{\mathrm{b}}(\psi)\right),
\end{aligned}
$$

with $\left(r_{\mathrm{a}}(\psi), z_{\mathrm{a}}(\psi)\right)$ the magnetic axis where $\nabla \psi=0$ and $\psi$ has a local extremum in $\Omega_{\mathrm{L}}$, and $\left(r_{\mathrm{b}}(\psi), z_{\mathrm{b}}(\psi)\right)$ the coordinates of the point that determines the plasma boundary. The point $\left(r_{\mathrm{b}}, z_{\mathrm{b}}\right)$ is either an X-point of $\psi$ or the contact point with the limiter $\Gamma_{L}$.

-Domains $\Omega_{\mathrm{c}_{i}}$ represent the poloidal field coils carrying currents

$$
j_{\phi}=\frac{I_{i}}{S_{i}}
$$

where $S_{i}$ is the section area of the coil and $I_{i}$ is the current flowing in the coil. In the static modelization $I_{i}$ is a constant whereas in the quasi-static case it relates to voltages $v_{j}(t)$ in the suppliers and to self and mutual induction

$$
I_{i}(t)=\sum_{j=1}^{N_{s}} \boldsymbol{S}_{i j} v_{j}(t)+\sum_{j=1}^{N_{c}} \boldsymbol{R}_{i j} \frac{1}{S_{j}} \int_{\Omega_{\mathrm{c}_{j}}} \frac{\partial \psi}{\partial t} d r d z, \quad 1 \leq i \leq N_{c}
$$

via electric circuit equations (see [3] Appendix A and [6] Appendix B). Here $N_{s}$ is the number of suppliers, $N_{c}$ is the number of coils, matrix $\boldsymbol{S}$ has dimension $N_{c} \times N_{s}$ and matrix $\boldsymbol{R}$ has dimension $N_{c} \times N_{c}$. In the simplest case where each circuit is composed of one coil and one voltage supplier, $\boldsymbol{S}$ and $\boldsymbol{R}$ are diagonal with $\boldsymbol{S}_{i i}=\frac{n_{i}}{R_{i}}$ and $\boldsymbol{R}_{i i}=-\frac{2 \pi n_{i}^{2}}{R_{i}}, n_{i}$ being the number of turns and $R_{i}$ the resistance. 
-Domain $\Omega_{\mathrm{ps}}$ represents passive structures. The expression for $j_{\phi}$ in these domains depends on the modelization considered. For static equilibrium $j_{\phi}=$ 0 is usually considered whereas for quasi-static evolution of the equilibrium

$$
j_{\phi}=-\frac{\sigma}{r} \frac{\partial \psi}{\partial t}
$$

where $\sigma$ is the conductivity of the passive structure.

Equilibrium equation (3). can either be considered in the whole poloidal plane $\Omega_{\infty}$ with $\psi=0$ as boundary condition at infinity and on the $r=0$ axis, or on a restricted bounded domain (whose boundary can be viewed as a measurement contour $\Gamma$ and is illustrated by the green dashed line on Figure 1). Both type of problems are addressed in NICE, the first one for the direct and inverse static and quasi-static modes and the second one for the equilibrium reconstruction mode.

\subsection{FEM discretized operators and derivatives}

For the equilibrium reconstruction mode as well as the direct and inverse modes of NICE equation (3) is discretized using a P1 finite element method based on a triangular mesh [19, 12, 3]. In this Section we provide some details on the discretized operators common to different modes of NICE as well as on the computation of their derivatives which are used in the Newton and SQP algorithms implemented for the resolution of non-linear direct and inverse problems. More operators specific to a given mode are presented later in the document.

There are two possible choices for the spatial domain to be triangulated. It can either be a restricted domain enclosed in the measurement contour $\Gamma$ or a sufficiently large semi-circle centered at the origin and containing the whole geometry of the tokamak. The first case is called the bounded domain case (see Figure 2) and the second the ABB domain case (see Figures 3 and 4). ABB stands for Albanase, Blum, de Barbieri [20] who first introduced the boundary integral method on the semi-circle used to take into account conditions at infinity. We refer to [3] for its precise expression and to [21, Chapter 2.4] for details on its derivation. Alternative approaches for the incorporation of boundary conditions at infinity were more recently presented in [22].

Let us denote by $\lambda_{i}(r, z)$ the $N$ Lagrangian basis functions associated to the inner vertices of the mesh and by $\lambda_{d, i}$ the $N_{b}$ ones associated to boundary 


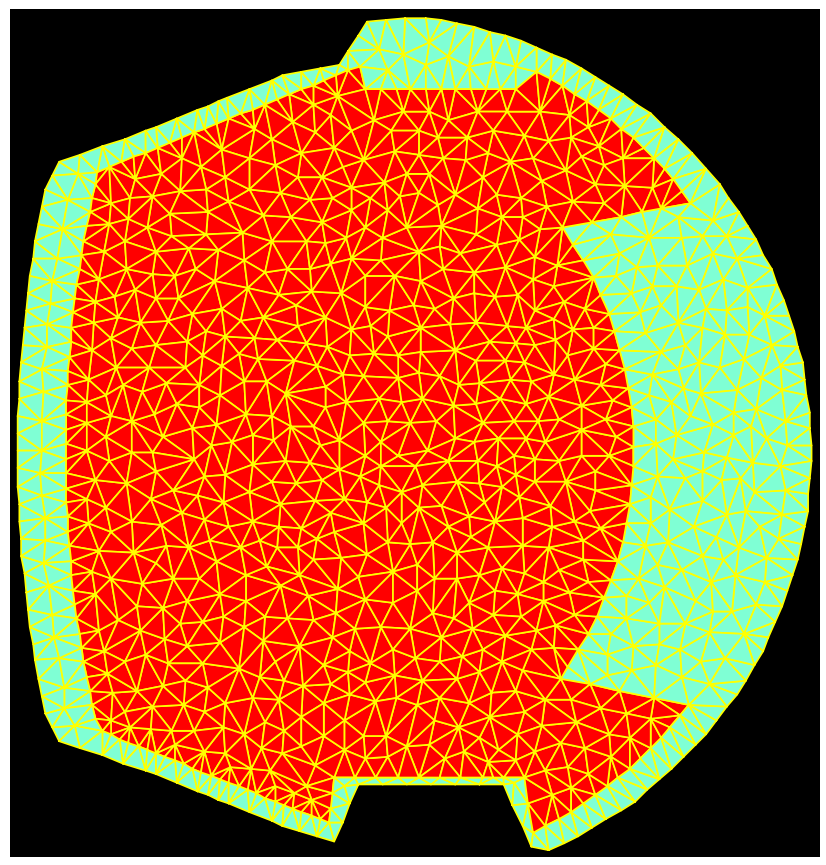

Figure 2: Example mesh for WEST tokamak in the bounded domain case. In red is the region inside the limiter. 


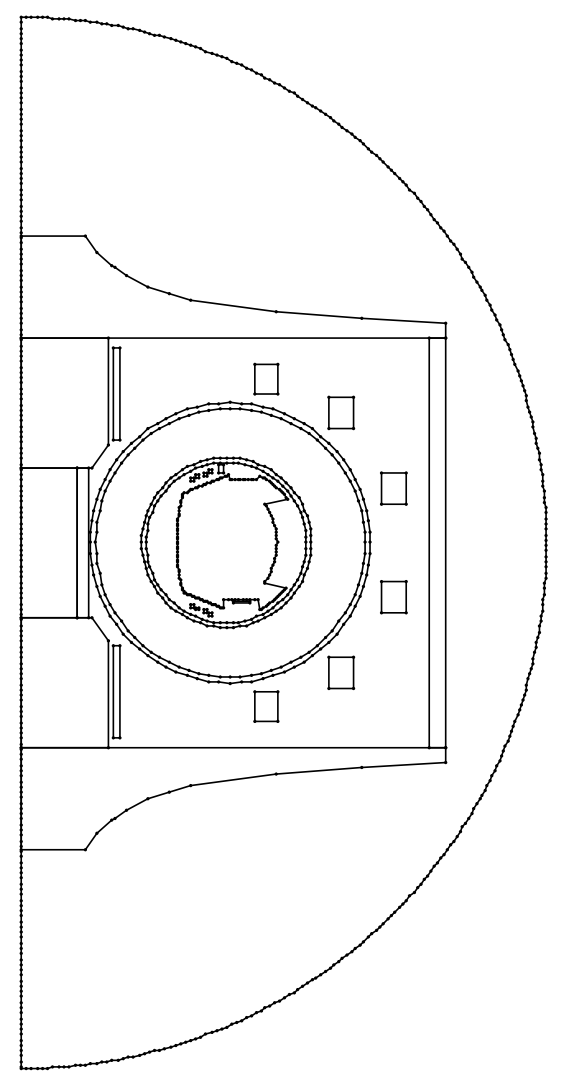

Figure 3: The ABB domain for WEST tokamak. 


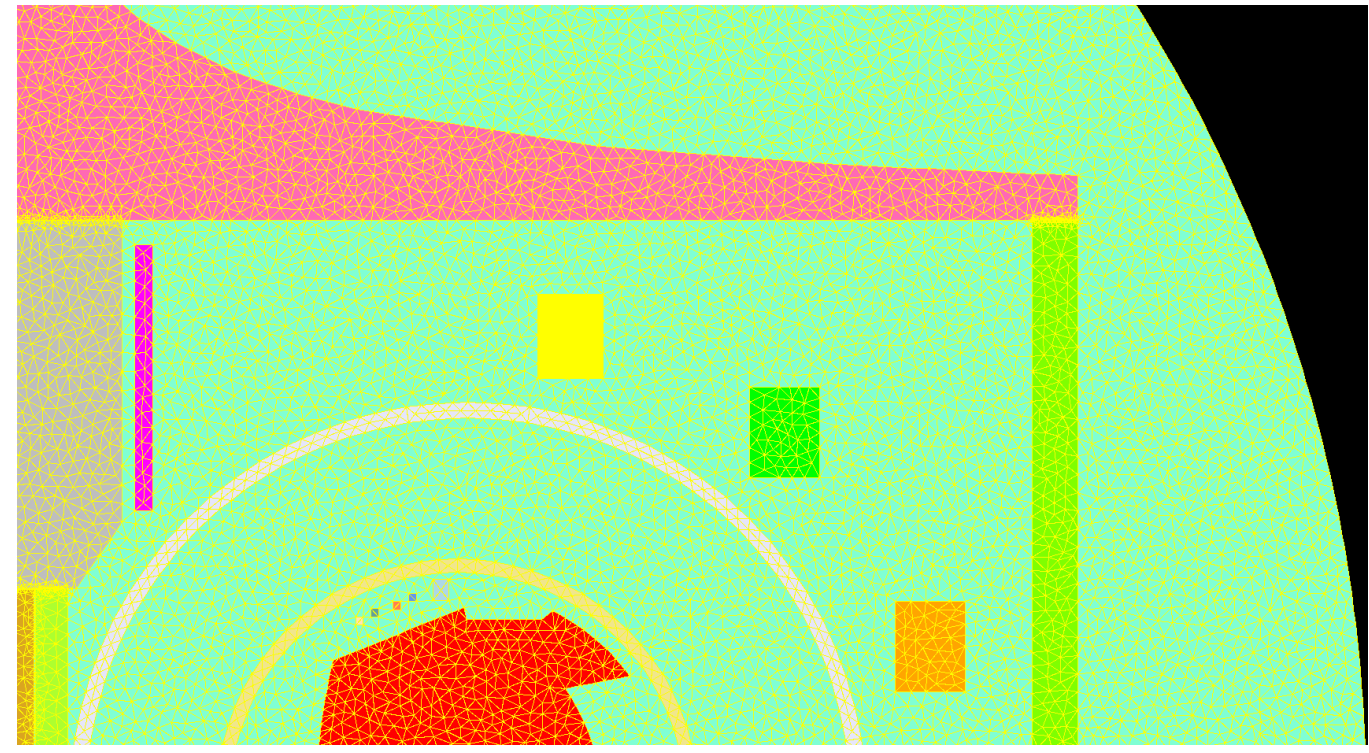

Figure 4: Zoom on an example mesh in the ABB domain case for WEST tokamak. Different parts of the machine appear in different colors (iron structure, PF coils, vacuum vessel, limiter).

vertices where Dirichlet conditions are applied $(r=0$ in the ABB case or $\Gamma$ in the bounded domain case).

Approximating the poloidal flux by $\psi_{h}=\sum_{i=1}^{N} \psi_{i} \lambda_{i}(r, z)+\sum_{i=1}^{N_{b}} \psi_{d, i} \lambda_{d, i}(r, z)$ and testing equation (3) against every inner basis function leads to the definition of the following matrices and vectors:

state variable. $\boldsymbol{\psi}$ denotes the vector of $N$ finite elements coefficients $\psi_{i}$

elliptic operator. split into a linear and a non linear part (if iron is present), $\boldsymbol{A} \boldsymbol{\psi}+\boldsymbol{A}_{\mu}(\boldsymbol{\psi})$. Matrix $\boldsymbol{A}$ is of size $N \times N$ and coefficients

$$
\boldsymbol{A}_{i j}=\int_{\Omega-\Omega_{\mathrm{f}}} \frac{1}{\mu_{0} r} \nabla \lambda_{i} \nabla \lambda_{j} d r d z
$$

involving the scalar product of the gradients of basis functions $i$ and $j$. Vector $\mathbf{A}_{\mu}(\boldsymbol{\psi})$ of dimension $N$ has coefficients

$$
\mathbf{A}_{\mu, i}(\boldsymbol{\psi})=\int_{\Omega_{\mathrm{f}}} \frac{1}{\mu\left(\psi_{h}\right) r} \nabla \lambda_{i} \nabla \psi_{h} d r d z
$$


The integrals are computed as sums of integrals over the triangles $T$ of the mesh on each of which the barycentric quadrature rule is used for the integral approximation. The Jacobian $\mathbf{A}_{\mu, \boldsymbol{\psi}}(\boldsymbol{\psi})$ is computed as

$$
\left[\mathbf{A}_{\mu, \psi}\right]_{i j}=\int_{\Omega_{\mathrm{f}}} \frac{\nabla \lambda_{i} \nabla \lambda_{j}}{\mu\left(\psi_{h}\right) r}-\frac{2 \mu_{f}^{\prime}\left(\left|\nabla \psi_{h}\right|^{2} r^{-2}\right)}{\mu_{f}^{2}\left(\left|\nabla \psi_{h}\right|^{2} r^{-2}\right) r^{3}}\left(\nabla \lambda_{i} \nabla \psi_{h}\right)\left(\nabla \lambda_{j} \nabla \psi_{h}\right) d r d z
$$

boundary conditions. Dirichlet boundary conditions, a vector $\boldsymbol{\psi}_{d}$ of size $N_{b}$ number of vertices on the boundary, are applied using matrix $\boldsymbol{A}_{d}$ of size $N \times N_{b}$ with coefficients

$$
\boldsymbol{A}_{d, i j}=\int_{\Omega} \frac{1}{\mu_{0} r} \nabla \lambda_{i} \nabla \lambda_{d, j} d r d z
$$

This matrix is only used in the bounded domain case since for the ABB domain case, $\boldsymbol{\psi}_{d}=0$.

In the ABB domain case the boundary integral method on the semi-circle leads to the definition of a matrix $\boldsymbol{C}$ of size $N \times N$ (see [3]).

plasma current density. $\mathbf{J}\left(\boldsymbol{\psi}, \lambda, \mathbf{u}_{\mathcal{A}}, \mathbf{u}_{\mathcal{B}}\right)$ vector of size $N$ and coefficient

$$
\mathbf{J}\left(\boldsymbol{\psi}, \lambda, \mathbf{u}_{\mathcal{A}}, \mathbf{u}_{\mathcal{B}}\right)_{i}=\int_{\Omega_{\mathrm{p}}\left(\psi_{h}\right)} \lambda\left(\frac{r}{r_{0}} \mathcal{A}\left(\psi_{\mathrm{N}}\right)+\frac{r_{0}}{r} \mathcal{B}\left(\psi_{\mathrm{N}}\right)\right) \lambda_{i} d r d z
$$

Functions $\mathcal{A}$ and $\mathcal{B}$ are decomposed in a basis of functions $\phi_{i}$ defined on $[0,1]$. In NICE piecewise linear and cubic splines functions are implemented.

$$
\mathcal{A}(x)=\sum_{i=1}^{N_{\mathcal{A}}} u_{A i} \phi_{i}(x), \quad \mathcal{B}(x)=\sum_{i=1}^{N_{\mathcal{B}}} u_{B i} \phi_{i}(x),
$$

and $\mathbf{u}_{A}, \mathbf{u}_{B}$ denote the vectors of degrees of freedom of $\mathcal{A}$ and $\mathcal{B}$ in the chosen decomposition basis. In the direct and inverse modes of NICE functions $\mathcal{A}$ and $\mathcal{B}$ can also have a parametric representation of the type

$$
\mathcal{A}(x)=\beta\left(1-x^{\alpha}\right)^{\gamma}, \quad \mathcal{B}(x)=(1-\beta)\left(1-x^{\alpha}\right)^{\gamma}
$$

The mesh does not resolve the boundary of plasma domain $\Omega_{\mathrm{p}}\left(\psi_{h}\right)$ and a quadrature rule needs to be specified for integrals over intersections of mesh triangles with the plasma domain $T \cap \Omega_{\mathrm{p}}\left(\psi_{h}\right)$. Barycenter quadrature is used but the quadrature point and weight depend non linearly on $\psi_{h}$. This needs 
to be taken into account in the computation of the $\operatorname{Jacobian} \mathbf{J}_{\boldsymbol{\psi}}\left(\boldsymbol{\psi}, \lambda, \mathbf{u}_{\mathcal{A}}, \mathbf{u}_{\mathcal{B}}\right)$. This differentiation is not straightforward and technical details are given in [3. The computation of Jacobians $\mathbf{J}_{\mathbf{u}_{\mathcal{A}}}\left(\boldsymbol{\psi}, \lambda, \mathbf{u}_{\mathcal{A}}, \mathbf{u}_{\mathcal{B}}\right)$ and $\mathbf{J}_{\mathbf{u}_{\mathcal{B}}}\left(\boldsymbol{\psi}, \lambda, \mathbf{u}_{\mathcal{A}}, \mathbf{u}_{\mathcal{B}}\right)$ which are needed for equilibrium reconstruction on the other hand does not rise any particular difficulty.

Finally we will use the notation

$$
J_{p}\left(\boldsymbol{\psi}, \mathbf{u}_{\mathcal{A}}, \mathbf{u}_{\mathcal{B}}\right)=\int_{\Omega_{\mathrm{p}}\left(\psi_{h}\right)}\left(\frac{r}{r_{0}} \mathcal{A}\left(\psi_{\mathrm{N}}\right)+\frac{r_{0}}{r} \mathcal{B}\left(\psi_{\mathrm{N}}\right)\right) d r d z
$$

such that the total plasma current is computed as

$$
I_{p}=\lambda J_{p}\left(\boldsymbol{\psi}, \mathbf{u}_{\mathcal{A}}, \mathbf{u}_{\mathcal{B}}\right)
$$

PF coils. matrix $\boldsymbol{L}$ of size $N \times N_{c}$ and vector $\mathbf{u}_{I}$ of size $N_{c}$ holding the currents $I_{i}$ of the $N_{c}$ coils contained in the domain under consideration (all coils in the ABB domain case but not in the bounded domain case), with coefficients

$$
\boldsymbol{L}_{i j}=\frac{1}{S_{j}} \int_{\Omega_{\mathrm{c}_{j}}} \lambda_{i} d r d z
$$

such that the PF coils term in the static case is $\boldsymbol{L} \mathbf{u}_{I}$ and in the quasi-static evolution case is $\boldsymbol{L} \boldsymbol{S} \mathbf{v}+\boldsymbol{L} \boldsymbol{R} \boldsymbol{L}^{T} \frac{d \boldsymbol{\psi}}{d t}$ with $\mathbf{v}$ the vector of the $N_{s}$ voltages passive structures. matrix $\boldsymbol{J}_{p s}$ of size $N \times N$, associated to the inductive terms in the passive structures with coefficients

$$
\left[\boldsymbol{J}_{p s}\right]_{i j}=-\sum_{k=1}^{N_{p s}} \int_{\Omega_{\mathrm{ps}_{k}}} \frac{\sigma_{k}}{r} \lambda_{i} \lambda_{j} d r d z
$$

where $N_{p s}$ is the number of passive structure and $\sigma_{k}$ their conductivity.

\subsection{Newton and PDE-constrained optimization algorithms}

With the matrix and vector operators defined in the previous section different direct and inverse problems can be formulated in NICE and are detailed in the remaining sections of this document. Here we present the main algorithms using a generic formulation. We will define different model equations e, state $\mathbf{y}$ and control $\mathbf{u}$ variables as well as cost functions $J(\mathbf{y}, \mathbf{u})$. They will differ from one mode to the other in the code but the resolution algorithms are the same. 
The direct problem formulation. is:

$$
\text { find } \mathbf{y} \text { such that } \mathbf{e}(\mathbf{y}, \mathbf{u})=0 \text {, }
$$

where $\mathbf{u}$ is here a constant parameter.

The inverse problem formulation. is:

$$
\min _{\mathbf{y}, \mathbf{u}} J(\mathbf{y}, \mathbf{u}) \quad \text { under the constraint } \mathbf{e}(\mathbf{y}, \mathbf{u})=0 \text {, }
$$

Throughout this document several explicit formulations are given for $\mathbf{e}$ and $J$. As a starter and to make it more concrete let us consider the static equilibrium problem in the $\mathrm{ABB}$ domain detailed in Section 5. The sate variable is $\mathbf{y}:=\boldsymbol{\psi}$, the control variable is $\mathbf{u}:=\mathbf{u}_{I}$ the currents in the coils and $p^{\prime}$ and $f f^{\prime}$ are given (thus $\mathbf{u}_{\mathcal{A}}, \mathbf{u}_{\mathcal{B}}$ and $\lambda$ are fixed parameters). The direct model in ABB domain is

$$
\mathbf{e}(\mathbf{y}, \mathbf{u}):=(\boldsymbol{A}+\boldsymbol{C}) \boldsymbol{\psi}+\mathbf{A}_{\mu}(\boldsymbol{\psi})-\mathbf{J}\left(\boldsymbol{\psi}, \lambda, \mathbf{u}_{\mathcal{A}}, \mathbf{u}_{\mathcal{B}}\right)-\boldsymbol{L} \mathbf{u}_{I}=0
$$

and the cost function is

$$
J(\mathbf{y}, \mathbf{u})=\frac{1}{2}\|\boldsymbol{K} \mathbf{y}\|^{2}+\frac{1}{2}\|\boldsymbol{R u}\|^{2}
$$

with a quadratic misfit term, imposing a levelset of the poloidal flux to go through a set of desired points, and a regularization term (see Section 5).

In NICE direct problems are solved thanks to Newton's method. If we denote the Newton increment by $\Delta \mathbf{y}=-\mathbf{e}_{\mathbf{y}}^{-1}\left(\mathbf{y}^{n}, \mathbf{u}\right) \mathbf{e}\left(\mathbf{y}^{n}, \mathbf{u}\right)$ this iterative algorithm reads:

$$
\mathbf{y}^{n+1}=\mathbf{y}^{n}+\Delta \mathbf{y}
$$

Inverse problems are solved thanks to a quasi-SQP method with reduced Hessian (QSQP). SQP methods are well documented [23, 24] and a clear summary is found in [6, Appendix A]. An SQP method can be seen as a Newton method to solve the non-linear system given by the fisrt order optimality condition for the Lagrangian of the PDE-constrained optimization problem

$$
\mathcal{L}(\mathbf{y}, \mathbf{u}, \mathbf{p})=J(\mathbf{y}, \mathbf{u})+(\mathbf{p}, \mathbf{e}(\mathbf{y}, \mathbf{u}))
$$

where vector $\mathbf{p}$ of size $N$ is the adjoint variable or Lagrange multiplier for the constraint of the model. This first method also known as Newton-Lagrange 
method is implemented in NICE and was used in [4] for equilibrium reconstruction. It was also already used in [3] for the static inverse problem (presented below in Section 5). The drawback of this method is that it demands the resolution of linear systems of size $\left(2 N+N_{u}\right)$, where $N$ is the dimension of the state and adjoint variables and $N_{u}$ the dimension of the control. When $N$ is large this can become time consumming and an excellent alternative is the SQP formulation with reduced Hessian which is intensively used in NICE and was already used in [6] for the quasi-static inverse problem (see Section 5.2). It is the following two-step iterative algorithm.

quasi $S Q P$ with reduced Hessian algorithm (QSQP):.

1. control variable update step

$$
M\left(\mathbf{u}^{n+1}-\mathbf{u}^{n}\right)=-\mathbf{m}
$$

2. state variable update step

$$
\mathbf{y}^{n+1}=\mathbf{y}^{n}+\Delta \mathbf{y}+\boldsymbol{S}\left(\mathbf{u}^{n+1}-\mathbf{u}^{n}\right)
$$

where

$$
\begin{gathered}
\Delta \mathbf{y}=-\mathbf{e}_{\mathbf{y}}^{-1}\left(\mathbf{y}^{n}, \mathbf{u}^{n}\right) \mathbf{e}\left(\mathbf{y}^{n}, \mathbf{u}^{n}\right) \\
\boldsymbol{S}=-\mathbf{e}_{\mathbf{y}}^{-1}\left(\mathbf{y}^{n}, \mathbf{u}^{n}\right) \mathbf{e}_{\mathbf{u}}\left(\mathbf{y}^{n}, \mathbf{u}^{n}\right) \\
\boldsymbol{M}=J_{\mathbf{u u}}\left(\mathbf{y}^{n}, \mathbf{u}^{n}\right)+\boldsymbol{S}^{T} J_{\mathbf{y u}}\left(\mathbf{y}^{n}, \mathbf{u}^{n}\right)+J_{\mathbf{u y}}\left(\mathbf{y}^{n}, \mathbf{u}^{n}\right) \boldsymbol{S}+\boldsymbol{S}^{T} J_{\mathbf{y y}}\left(\mathbf{y}^{n}, \mathbf{u}^{n}\right) \boldsymbol{S}
\end{gathered}
$$

and

$$
\mathbf{m}=J_{\mathbf{u}}^{T}\left(\mathbf{y}^{n}, \mathbf{u}^{n}\right)+\boldsymbol{S}^{T} J_{\mathbf{y}}^{T}\left(\mathbf{y}^{n}, \mathbf{u}^{n}\right)+J_{\mathbf{u y}}\left(\mathbf{y}^{n}, \mathbf{u}^{n}\right) \Delta \mathbf{y}+\boldsymbol{S}^{T} J_{\mathbf{y y}}\left(\mathbf{y}^{n}, \mathbf{u}^{n}\right) \Delta \mathbf{y}
$$

At each iteration this algorithm demands the resolution of $N_{u}+1$ linear systems (17)-(18) of size $N$ involving the same matrix $\mathbf{e}_{\mathbf{y}}\left(\mathbf{y}^{n}, \mathbf{u}^{n}\right)$ with different righ-hand sides which can be done very efficiently and of one smaller linear system of size $N_{u}$ in 15 .

One can notice that second order derivatives appear only for the cost function and not for the model equation in (19) and (20). This is due to the fact that on the one hand the model equation in our applications is affine in the control variable, $\mathbf{u}$, therefore has 0 second order derivative, and on 
the other hand we want to avoid the expensive computation of other second order derivatives of $\mathbf{J}\left(\boldsymbol{\psi}, \lambda, \mathbf{u}_{\mathcal{A}}, \mathbf{u}_{\mathcal{B}}\right)$ and neglect them. Neglecting these second order derivatives of the model equation also allows to avoid having to compute the adjoint state variable. Moreover in some cases where in the cost function the observation operator is non-linear we also neglect some of the second order derivatives terms in $J_{\mathbf{y u}}, J_{\mathbf{u y}}$ and $J_{\mathbf{y y}}$ (see equilibrium reconstruction with internal measurements in Section 4.2). NICE uses a quasi SQP algorithm and not an exact SQP algorithm. The excellent convergence properties of the code allow us to think that these modifications are not an issue for the applications specific to tokamaks dealt with in NICE.

It should also be noted that if one neglects the first order derivative $\mathbf{J}_{\psi}$ term in the computation of model derivative $\mathbf{e}_{\mathbf{y}}$ this algorithm applied to the equilibrium reconstruction problem of Section 4 is exactly the original algorithm from the code EQUINOX. In the original EQUINOX paper [2] it is described as a fixed-point method to solve the model equation in $\boldsymbol{\psi}$ for which the control variable $\mathbf{u}$ is updated at each iteration. It appears clearly that this method is another (more approximated) quasi SQP with reduced Hessian method. In our experience however this method does not always demonstrates the same quality and speed of convergence as the one used in NICE which involves more derivative terms.

\subsection{Cost functions notation}

Throughout this document cost functions of the following type appear

$$
J(\boldsymbol{\psi}, \mathbf{u})=\frac{1}{2}\|\mathbf{M}(\boldsymbol{\psi}, \mathbf{u})-\mathbf{m}\|^{2}+\frac{1}{2}\|\boldsymbol{R} \mathbf{u}\|^{2}
$$

The first term is the data misfit term and the second the regularization term.

Each measurement data $m_{i}$ is a given quantity which goes together with a given absolute measurement error, abserr, and an relative measurement error, relerr, and we form the quantity $\sigma_{i}=\max \left(\right.$ abserr, relerr $\left.\left|m_{i}\right|\right)$. All vectorial quantities related to measurements which appear in cost functions are normalized quantities. The vector $\mathbf{m}$ of measurements has components $\mathbf{m}_{i}=m_{i} / \sigma_{i}$ and the observation operator $\mathbf{M}(\boldsymbol{\psi}, \mathbf{u})$ computes from the model the equivalent of these normalized measured quantities. In NICE absolute and relative errors can either be provided together with the used data set (for example these quantities should be provided in IMAS) or set by the user through input code parameters. 
Regularization terms are needed due to the ill-posedness of the inverse problems under consideration and they guarantee smoothness of the identified quantities. Regularization and penalization parameters are included in $\boldsymbol{R}$ matrices and are provided by the user through input code parameters.

\section{NICE magnetic measurements interpolation and plasma bound- ary reconstruction mode}

The goal of this section is to present the magnetic measurements interpolation and plasma boundary reconstruction mode of NICE.

\subsection{Method}

Toroidal harmonics are used to represent the poloidal flux in an annular domain surrounding the plasma. In such a domain $\psi$ satisfies

$$
-\Delta^{*} \psi=0
$$

and can be expanded in a series of toroidal harmonics (see [1] and references therein).

This method allows a fast fit to magnetic measurements and the poloidal field function can then be used to compute the plasma boundary. It allows also to compute precisely the poloidal flux and its gradient on any contour surrounding the plasma and thus on $\Gamma$ the measurements contour in NICE bounded domain case (green dashed line in Figure 1). These Cauchy conditions on $\Gamma,\left(g=\psi, h=\partial_{n} \psi\right)$, can then be used in a second step to reconstruct the full non-linear free-boundary Grad-Shafranov equilibrium problem inside the domain $\Omega$ limited by $\Gamma$. This is presented in the following Section 4 .

Below we propose to use a simple additional regularization term which was not originally present in [1]. This regularization operates directly on the toroidal harmonics expansion in order to obtain a smooth representation of flux even faraway from the measurements contour $\Gamma$ inside the domain $\Omega$.

The toroidal coordinates system [25, 26] or bipolar coordinates system (if we ignore the angular toroidal variable) $(\zeta, \eta) \in \mathbb{R}_{*}^{+} \times[0,2 \pi]$ about the pole $F_{p}=\left(r_{p}, z_{p}\right)$ is related to the cylindrical coordinates system $(r, z)$ by

$$
r=\frac{r_{p} \sinh \zeta}{\cosh \zeta-\cos \eta} \quad \text { and } z-z_{p}=\frac{r_{p} \sin \eta}{\cosh \zeta-\cos \eta}
$$

We assume that $F_{p}$ lies inside the plasma domain where the homogeneous equation, $-\Delta^{*} \psi=0$, is not satisfied. 
Following [1] the poloidal flux in the vacuum surrounding the plasma is written as $\psi=\psi_{C}+\psi_{t h}$. The term $\psi_{C}$ is the given contribution of the poloidal field coils $C_{k}$ with current density $j_{C_{k}}=\frac{I_{k}}{S_{k}}$ evaluated thanks to the free space Green function:

$$
\psi_{C}(x)=\sum_{k} \int_{C_{k}} j_{C_{k}} G(y, x) d y
$$

The toroidal harmonics expansion is represented by $\psi_{t h}$, sum of two terms $\psi_{\text {ex }}$ and $\psi_{\text {in }}$, corresponding respectively to external and internal harmonics:

$$
\left\{\begin{aligned}
\psi_{t h}= & \psi_{e x}+\psi_{i n}, \\
\psi_{e x}= & \frac{r_{p} \sinh \zeta}{\sqrt{\cosh \zeta-\cos \eta}} \times \\
& {\left[\sum_{n=0}^{e} a_{n}^{e} Q_{n-1 / 2}^{1}(\cosh \zeta) \cos (n \eta)+\sum_{n=1}^{n_{b}^{e}} b_{n}^{e} Q_{n-1 / 2}^{1}(\cosh \zeta) \sin (n \eta)\right], } \\
\psi_{i n}= & \frac{r_{p} \sinh \zeta}{\sqrt{\cosh \zeta-\cos \eta}} \times \\
& {\left[\sum_{n=0}^{n_{a}^{i}} a_{n}^{i} P_{n-1 / 2}^{1}(\cosh \zeta) \cos (n \eta)+\sum_{n=1}^{n_{b}^{i}} b_{n}^{i} P_{n-1 / 2}^{1}(\cosh \zeta) \sin (n \eta)\right], }
\end{aligned}\right.
$$

where $P_{n-1 / 2}^{1}$ and $Q_{n-1 / 2}^{1}$ are the associated Legendre functions of first and second kind, of degree one and half integer order [27], also called toroidal harmonics when evaluated at point $\cosh \zeta$. Functions $P_{n-1 / 2}^{1}$ have a singularity when $\zeta \rightarrow \infty$ that is to say at point $F_{p}$. On the contrary functions $Q_{n-1 / 2}^{1}$ are singular when $\zeta \rightarrow 0$ that is to say on the axis $r=0$.

We denote by $\mathbf{u}$ the vector of the unknown expansion coefficients

$$
\mathbf{u}=\left(a_{0}^{e}, \ldots, a_{n_{a}^{e}}^{e}, b_{1}^{e}, \ldots, b_{n_{b}^{e}}^{e}, a_{0}^{i}, \ldots, a_{n_{a}^{i}}^{i}, b_{1}^{i}, \ldots, b_{n_{b}^{i}}^{i}\right) .
$$

The optimal set of coefficients $\mathbf{u}_{\text {opt }}$ can be computed from the minimization of the least-square cost function

$$
J_{0}(\mathbf{u})=\frac{1}{2}\|\mathbf{M} \mathbf{u}-\mathbf{m}\|^{2}
$$

where $\mathbf{m}$ is the vector of (normalized) flux loops and Bprobes measurements corrected from their known $\psi_{C}$ contribution, and $\boldsymbol{M} \mathbf{u}$ the equivalent quantities computed from the toroidal harmonics expansion of Eq. (22). Here the 
rectangular matrix $\boldsymbol{M}$ depends on the choice of the pole of the coordinates system.

$J_{0}$ is quadratic in $\mathbf{u}$ and is minimized simply by solving the associated normal equation, $\boldsymbol{M}^{T} \boldsymbol{M} \mathbf{u}-\boldsymbol{M}^{T} \mathbf{m}=0$, to find the unique optimal set of coefficients $\mathbf{u}_{\text {opt }}$.

Assuming enough toroidal harmonics are used the resulting representation of the poloidal field is always precise on $\Gamma$ the measurements contour but might become highly disturbed when moving towards the pole $F_{p}$ which is a singular point for the internal harmonics. Hence let us introduce the regularized functional

$$
J(\mathbf{u})=J_{0}(\mathbf{u})+R(\mathbf{u})
$$

where the regularization term $R$ involves the second order tangential derivative of $\psi$ along a particular circle $C$ surrounding the pole $F_{p}$,

$$
R(\mathbf{u})=\frac{\varepsilon}{2} \int_{C}\left|\frac{d^{2} \psi_{t h}(\mathbf{u})}{d s^{2}}\right|^{2} d s
$$

and is quadratic in $\mathbf{u}$.

The following remark leads to an obvious choice for the circle $C$. In toroidal coordinates the curves of constant $\zeta$ are non-intersecting circles of different radii

$$
\left(r-r_{p} \operatorname{coth} \zeta\right)^{2}+\left(z-z_{p}\right)^{2}=\frac{r_{p}^{2}}{\sinh ^{2} \zeta}
$$

that surround the pole but are not concentric. The $\zeta=0$ curve corresponds to the $z$-axis $(r=0)$. As the magnitude of $\zeta$ increases, the radius of the circles decreases and their centers approach the pole $F_{p}$. We choose the circle $C$ to be the circle of a constant $\zeta_{0}$ value. This choice considerably simplifies the computation of the second order tangential derivative since it is only required to compute derivatives with respect to $\eta$ to obtain the expression of the regularization term.

The curvilinear abscissa along the constant- $\zeta_{0}$ circle is given by

$$
s(\eta)=\int_{-\pi}^{\eta} \frac{r_{p}}{\cosh \zeta_{0}-\cos t} d t
$$

The second order tangential derivative along the circle of $\psi$ seen as a function of $s$ is

$$
\frac{d^{2} \psi_{t h}}{d s^{2}}=\frac{\partial^{2} \psi_{t h}}{\partial \eta^{2}}\left(\eta, \zeta_{0}\right)\left(\frac{d \eta}{d s}\right)^{2}+\frac{\partial \psi_{t h}}{\partial \eta}\left(\eta, \zeta_{0}\right) \frac{d^{2} \eta}{d s^{2}}
$$


with

$$
\frac{d \eta}{d s}=\frac{\cosh \zeta_{0}-\cos \eta}{r_{p}} \text { and } \frac{d^{2} \eta}{d s^{2}}=\frac{\left(\cosh \zeta_{0}-\cos \eta\right) \sin \eta}{r_{p}^{2}}
$$

and we obtain the expression of the regularization term

$$
\begin{aligned}
\int_{C_{\zeta_{0}}}\left|\frac{d^{2} \psi_{t h}}{d s^{2}}\right|^{2} d s= & \int_{-\pi}^{\pi}\left[\frac{\partial^{2} \psi_{t h}}{\partial \eta^{2}}\left(\eta, \zeta_{0}\right)\left(\frac{\cosh \zeta_{0}-\cos \eta}{r_{p}}\right)^{2}\right. \\
& \left.+\frac{\partial \psi_{t h}}{\partial \eta}\left(\eta, \zeta_{0}\right) \frac{\left(\cosh \zeta_{0}-\cos \eta\right) \sin \eta}{r_{p}^{2}}\right]^{2}\left[\frac{r_{p}}{\cosh \zeta_{0}-\cos \eta}\right] d \eta
\end{aligned}
$$

The $\psi_{t h}$ derivatives with respect to $\eta$ can be easily explicitly computed from Eq. 22 and the integral computed using a standard quadrature method.

Let us conclude this section with the complete algorithm implemented in NICE.

\section{Algorithm.}

1. Initialization: prepare filaments description of PF coils, provide code parameters for number of toroidal harmonics, regularization parameters, test parameter $d$ (see point 4 below), points defining contour $\Gamma$, number $N_{p}$ of required plasma boundary points

2. Choose the pole of the coordinates system $F_{p}$. Default initial guess is $F_{p}=\left(r_{0}, 0\right)$.

Assemble and minimize cost function (24) to find $\mathbf{u}_{\text {opt }}$.

3. Compute the current center $F_{c}=\left(r_{c}, z_{c}\right)$ defined as moments of the plasma current density [28, 29] which can be precisely computed as integrals on the limiter contour $\Gamma_{L}$ at every point of which the flux and the poloidal magnetic field can be evaluated thanks to the representation $\psi=\psi_{t h}\left(\mathbf{u}_{o p t}\right)+\psi_{C}$,

$$
\begin{aligned}
I_{p} & =\int_{\Gamma_{L}} \frac{1}{\mu_{0}} B_{s} d s \\
z_{c} I_{p} & =\int_{\Gamma_{L}} \frac{1}{\mu_{0}}\left(-r \log r B_{n}+z B_{s}\right) d s \\
r_{c}^{2} I_{p} & =\int_{\Gamma_{L}} \frac{1}{\mu_{0}}\left(2 r z B_{n}+r^{2} B_{s}\right) d s
\end{aligned}
$$

where $B_{s}$ (resp. $B_{n}$ ) denotes the poloidal field tangent (resp. normal) to the integration contour $\Gamma_{L}$. 
4. If $\left\|F_{c}-F_{p}\right\|>d$ set $F_{p}:=F_{c}$, re-assemble and minimize cost function (24) again, since matrix $\boldsymbol{M}$ in (23) depends on the pole of the coordinates system, to find a new $\mathbf{u}_{\text {opt }}$.

5. Output Cauchy conditions $\left(\psi, \partial_{n} \psi\right)$ on measurement contour $\Gamma$ and/or compute plasma boundary with the following algorithm:

- find $P_{b}$ the point defining the plasma boundary $\psi_{b}$ value. Either a limiter point or an X-point found thanks to a quasi Newton method for $\nabla \psi=0$

- starting from the $\left[F_{c}, P_{b}\right)$ ray define $N_{p}-1$ rays $\left[F_{c}, P_{k}\right)$ with a rotation of $\Delta \theta=2 \pi /\left(N_{p}-1\right)$ between each of them. Use Newton method along each ray to find the plasma boundary point where $\psi(r, z)-\psi_{b}=0$

\subsection{Numerical examples}

This mode of NICE also known as VacTH is routinely used at WEST for plasma shape identification and control during a shot [7]. Here is provided a typical example of plasma boundary reconstruction for a WEST-like equilibrium. The poloidal flux is computed using 24 toroidal harmonics for $\psi_{t h}$ (15 external harmonics with $n_{a}^{e}=n_{b}^{e}=7$ and 9 internal harmonics with $\left.n_{a}^{i}=n_{b}^{i}=4\right)$ and 24 filaments of currents modelizing the given current density in the PF coils for $\psi_{C}$. Figure 5 shows the fit to Bprobes measurements with a resulting root mean square error of $2 \mathrm{mT}$.

The $\zeta$ value defining the regularization circle is chosen such that the radius of this circle is $a\left(r_{\text {limiterMax }}-r_{\text {limiterMin }}\right)$ where $a=0.1$ is a code parameter. Another code parameter provides the value of regularization parameter which is here set to $\varepsilon=0.001$. A 60 points plasma boundary is computed by the method described above and is shown on Figure 6.

The algorithm is not machine dependent and was successfully applied to other tokamaks such as JET, TCV, AUG or also ISTTOK [30]. Figure 7 shows a JET-like equilibrium example and Figure 8 a TCV-like equilibrium example.

\section{NICE equilibrium reconstruction mode}

The goal of this section is to introduce the algorithm implemented in NICE for equilibrium reconstruction. Basic measurements used for equilibrium reconstruction are magnetic measurements but NICE can also use 


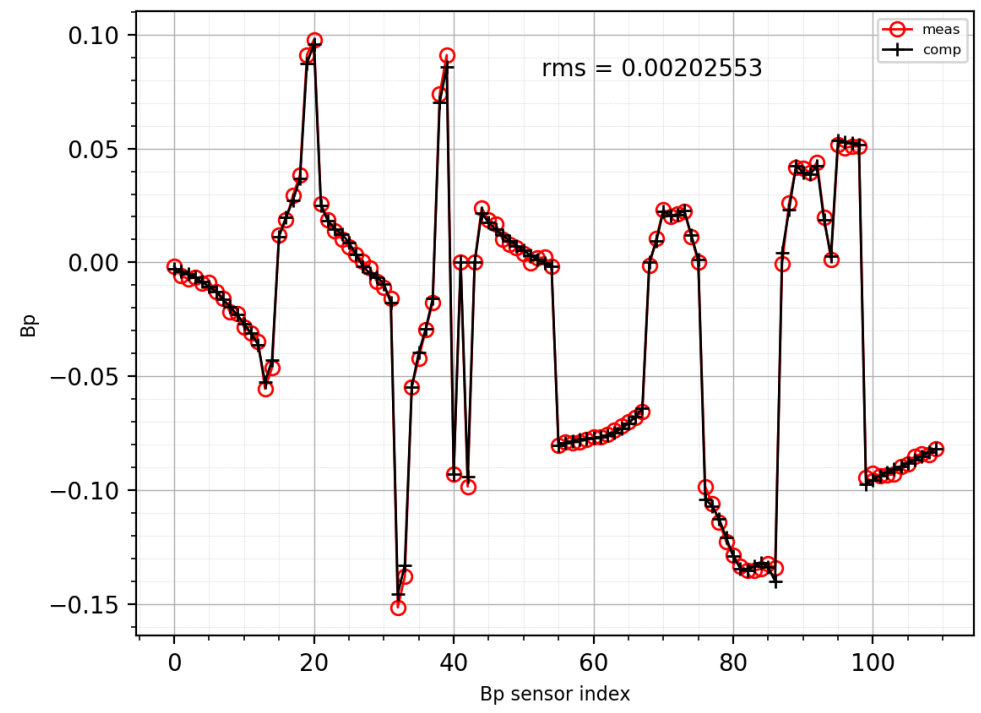

Figure 5: Measured and computed Bprobes values for a WEST-like plasma boundary reconstruction.

interferometry, polarimetry, motional Stark effect (MSE) and pressure measurements. These are introduced in Section 4.2 and we start by using magnetics only for simplicity.

\subsection{Problem formulation on a bounded domain with magnetic measurements}

Here magnetic measurements are in fact Cauchy data $\left(\psi, \frac{\partial \psi}{\partial n}\right)$ provided on the measurement contour $\Gamma$. These data are usually computed from a first call to NICE magnetics interpolation mode described in Section 3 but can also be provided by another code specific to a Tokamak which computes the poloidal flux outside the plasma such as XLOC at JET [31, 32].

Using the matrix notations of Sections 2.2, 2.3 and 2.4 Dirichlet data provide the $\boldsymbol{\psi}_{d}$ vector used in the resolution of the boundary value problem whereas Neumann data are considered as measurements and used in a cost function to be minimized to reconstruct the equilibrium. NICE uses $N_{b}$ values of $\frac{\partial \psi}{\partial n}$ given at the middle point of each segment of the mesh forming the $\Gamma$ contour.

We denote by $\mathbf{m}$ the vector of these (normalized) measurements and the equivalent quantities, $\boldsymbol{H} \boldsymbol{\psi}+\boldsymbol{H}_{d} \boldsymbol{\psi}_{d}$ computed from the FEM representation 


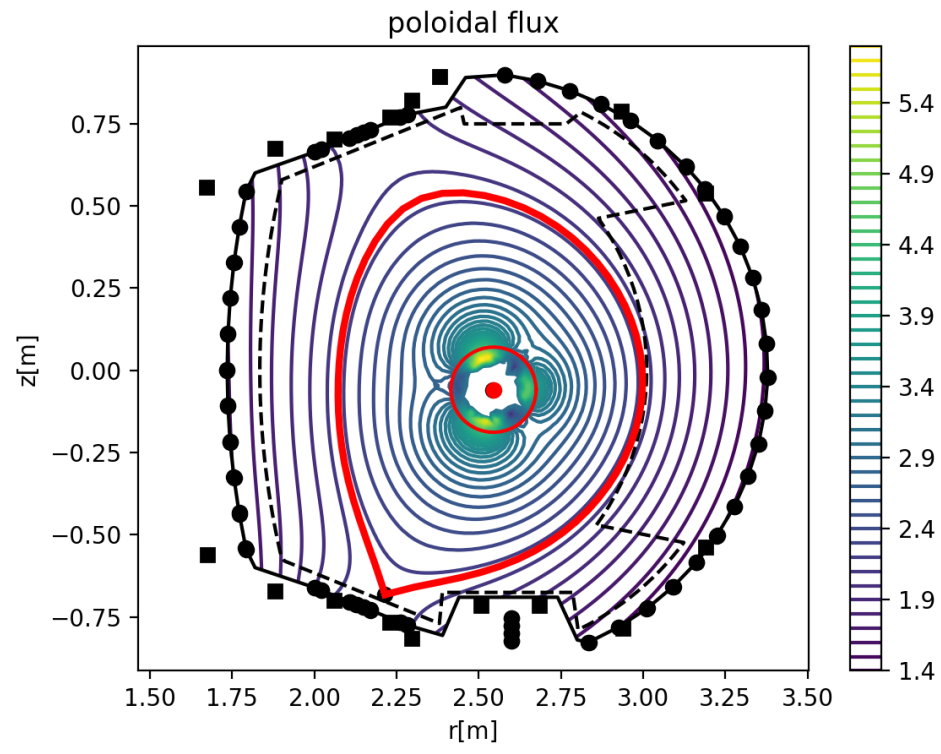

Figure 6: Poloidal flux $\psi=\psi_{t h}\left(\mathbf{u}_{o p t}\right)+\psi_{C}$ computed by a fit of toroidal harmonics to magnetic measurements for a WEST-like equilibrium. The measurement contour $\Gamma$ is shown in black, Bprobes are black circles and flux loops black squares. The limiter contour is the dashed black line. The plasma boundary with a low Xpoint is the thick red contour whereas the regularization circle is the thin red line surrounding the pole of the coordinate system. 


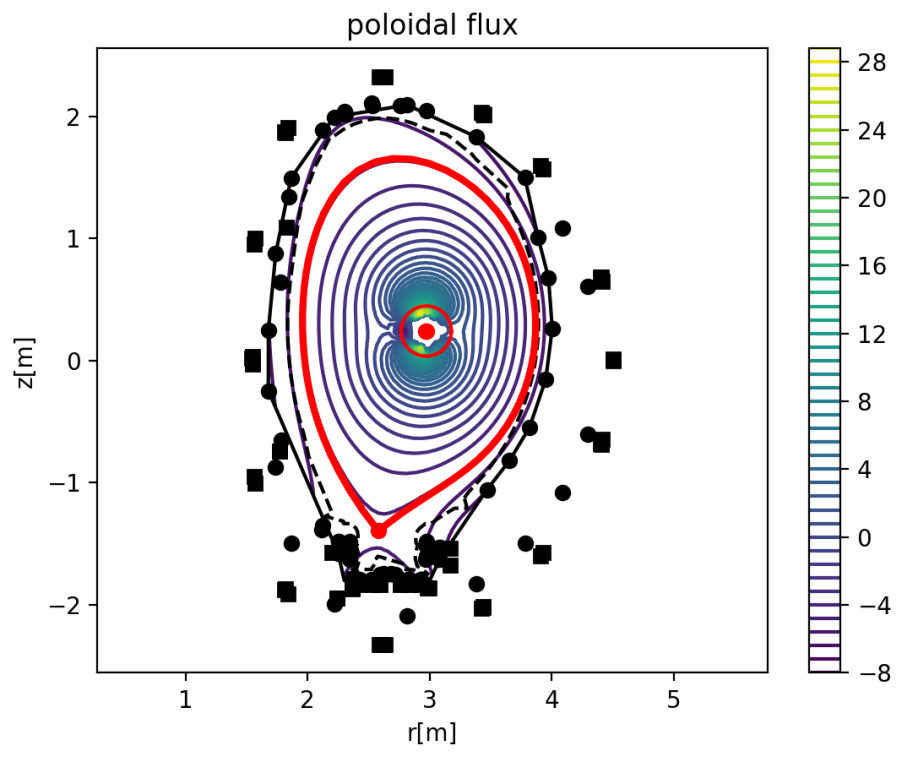

Figure 7: Poloidal flux $\psi=\psi_{t h}\left(\mathbf{u}_{o p t}\right)+\psi_{C}$ computed by a fit of toroidal harmonics to magnetic measurements for a JET-like equilibrium. The measurement contour $\Gamma$ is shown in black, Bprobes are black circles and flux loops black squares. The limiter contour is the dashed black line. The plasma boundary with a low Xpoint is the thick red contour whereas the regularization circle is the thin red line surrounding the pole of the coordinate system. 


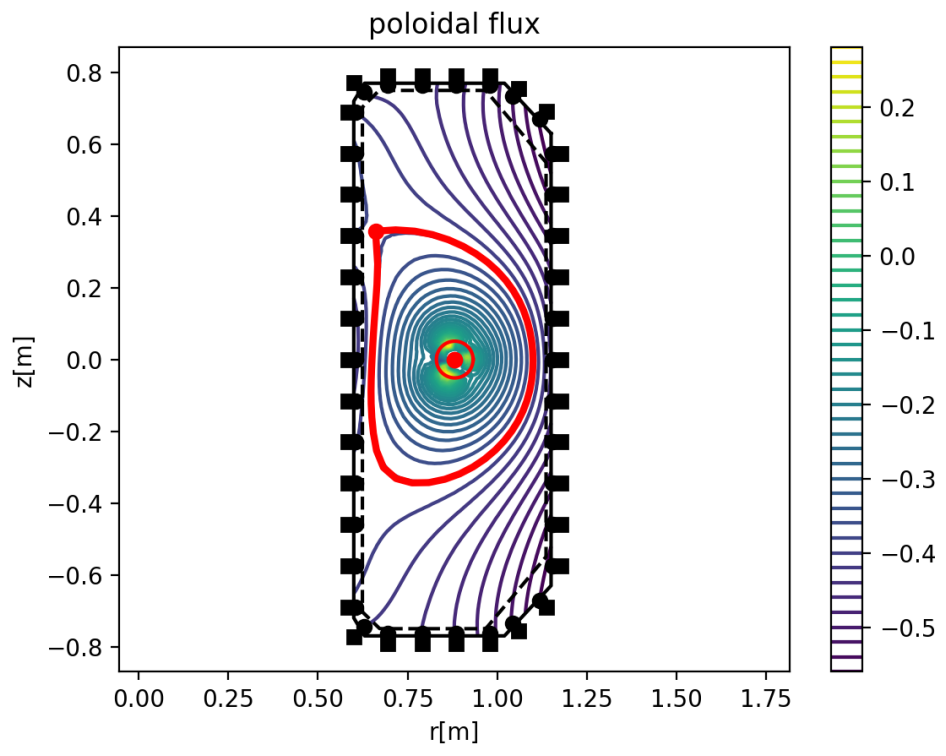

Figure 8: Poloidal flux $\psi=\psi_{t h}\left(\mathbf{u}_{o p t}\right)+\psi_{C}$ computed by a fit of toroidal harmonics to magnetic measurements for a TCV-like equilibrium. The measurement contour $\Gamma$ is shown in black, Bprobes are black circles and flux loops black squares. The limiter contour is the dashed black line. The plasma boundary with an upper Xpoint is the thick red contour whereas the regularization circle is the thin red line surrounding the pole of the coordinate system. 
of the poloidal flux thanks to the $N_{m} \times N$ matrix $\boldsymbol{H}$ and the $N_{m} \times N_{b}$ matrix $\boldsymbol{H}_{d}$.

The cost function, which depends on $\mathbf{y}:=\boldsymbol{\psi}$ and $\mathbf{u}:=\left(\mathbf{u}_{\mathcal{A}}, \mathbf{u}_{\mathcal{B}}\right)$ the degrees of freedom of functions $\mathcal{A}$ and $\mathcal{B}$, we consider is the following

$$
J(\mathbf{y}, \mathbf{u}):=\frac{1}{2}\left\|\boldsymbol{H} \boldsymbol{\psi}+\boldsymbol{H}_{d} \boldsymbol{\psi}_{d}-\mathbf{m}\right\|^{2}+\frac{1}{2}\left\|\boldsymbol{R}_{\mathcal{A}} \mathbf{u}_{\mathcal{A}}\right\|^{2}+\frac{1}{2}\left\|\boldsymbol{R}_{\mathcal{B}} \mathbf{u}_{\mathcal{B}}\right\|^{2}
$$

The first term on the right hand-side is the misfit term and the two others are regularization terms. They are the discrete expressions for

$$
\frac{\varepsilon_{\mathcal{A}}}{2} \int_{0}^{1}\left(\mathcal{A}^{\prime \prime}(x)\right)^{2} d x+\frac{\alpha_{\mathcal{A}}}{2}|\mathcal{A}(1)|^{2}
$$

and

$$
\frac{\varepsilon_{\mathcal{B}}}{2} \int_{0}^{1}\left(\mathcal{B}^{\prime \prime}(x)\right)^{2} d x+\frac{\alpha_{\mathcal{B}}}{2}|\mathcal{B}(1)|^{2}
$$

Parameters $\varepsilon$ enable to tune the smoothness of the identified functions whereas parameters $\alpha$ tune the penalization to zero of their value on the plasma boundary.

The free-boundary equilibrium model equation is

$$
\mathbf{e}(\mathbf{y}, \mathbf{u}):=\boldsymbol{A} \boldsymbol{\psi}+\boldsymbol{A}_{d} \boldsymbol{\psi}_{d}-\mathbf{J}\left(\boldsymbol{\psi}, \lambda, \mathbf{u}_{\mathcal{A}}, \mathbf{u}_{\mathcal{B}}\right)-\boldsymbol{L} \mathbf{u}_{I}=0
$$

and the equilibrium reconstruction problem is formulated as the folowing PDE-constrained optimization problem:

$$
\min _{\mathbf{y}, \mathbf{u}} J(\mathbf{y}, \mathbf{u}) \quad \text { such that } \mathbf{e}(\mathbf{y}, \mathbf{u})=0
$$

This problem is solved thanks to the QSQP algorithm given in Section 2.3. A first initialization step is used to set $\lambda$ in the current density term given the total plasma current $I_{p}$ which is either given with magnetic measurements or comes out from the toroidal harmonics procedure. Given initial guess $\boldsymbol{\psi}^{0}$, $\mathbf{u}_{\mathcal{A}}^{0}$ and $\mathbf{u}_{\mathcal{B}}^{0}, \lambda$ is chosen to satisfy

$$
I_{p}-\lambda J_{p}\left(\boldsymbol{\psi}^{0}, \mathbf{u}_{\mathcal{A}}^{0}, \mathbf{u}_{\mathcal{B}}^{0}\right)=0
$$

Using the notation of Section 2.3, the ingredients needed for the QSQP algorithm in equations (15), (16), (17), (18), (19) and (20) are 


$$
\begin{gathered}
\Delta \mathbf{y}=-\left(\boldsymbol{A}-\mathbf{J}_{\boldsymbol{\psi}}\left(\boldsymbol{\psi}^{n}, \lambda, \mathbf{u}_{\mathcal{A}}^{n}, \mathbf{u}_{\mathcal{B}}^{n}\right)\right)^{-1}\left(\boldsymbol{A} \boldsymbol{\psi}^{n}+\boldsymbol{A}_{d} \boldsymbol{\psi}_{d}-\mathbf{J}\left(\boldsymbol{\psi}^{n}, \lambda, \mathbf{u}_{\mathcal{A}}^{n}, \mathbf{u}_{\mathcal{B}}^{n}\right)-\boldsymbol{L} \mathbf{u}_{I}\right) \\
\boldsymbol{S}=-\left(\boldsymbol{A}-\mathbf{J}_{\boldsymbol{\psi}}\left(\boldsymbol{\psi}^{n}, \lambda, \mathbf{u}_{\mathcal{A}}^{n}, \mathbf{u}_{\mathcal{B}}^{n}\right)\right)^{-1}\left(\mathbf{J}_{\mathbf{u}}\left(\boldsymbol{\psi}^{n}, \lambda, \mathbf{u}_{\mathcal{A}}^{n}, \mathbf{u}_{\mathcal{B}}^{n}\right)\right) . \\
J_{\mathbf{y}}^{T}=\boldsymbol{H}^{T}\left(\boldsymbol{H} \boldsymbol{\psi}+\boldsymbol{H}_{d} \boldsymbol{\psi}_{d}-\mathbf{m}\right), \quad J_{\mathbf{u}}^{T}=\left[\begin{array}{c}
\boldsymbol{R}_{\mathcal{A}}^{T} \boldsymbol{R}_{\mathcal{A}} \mathbf{u}_{\mathcal{A}} \\
\boldsymbol{R}_{\mathcal{B}}^{T} \boldsymbol{R}_{\mathcal{B}} \mathbf{u}_{\mathcal{B}}
\end{array}\right] \\
J_{\mathbf{y y}}=\boldsymbol{H}^{T} \boldsymbol{H}, \quad J_{\mathbf{u u}}=\left[\begin{array}{cc}
\boldsymbol{R}_{\mathcal{A}}^{T} \boldsymbol{R}_{\mathcal{A}} & 0 \\
0 & \boldsymbol{R}_{\mathcal{B}}^{T} \boldsymbol{R}_{\mathcal{B}}
\end{array}\right], \quad J_{\mathbf{y u}}=0, \quad J_{\mathbf{u y}}=0
\end{gathered}
$$

\subsection{Internal measurements}

In addition to magnetics NICE can use internal measurements for equilibrium reconstruction.

\subsubsection{Interferometry and polarimetry}

Classical polarimetry. Polarimetry measurements in first approximation give the Faraday rotation of the angle of an infrared radiation crossing the section of the plasma along different lines of sight, $L^{i}$ :

$$
\alpha_{o b s}^{i} \approx \int_{L^{i}} c N_{e} \mathbf{B}_{p} \cdot \mathbf{t} d l
$$

where $c$ is a constant, $N_{e}$ is the electron density in the plasma and $\mathbf{t}$ is the unit vector tangent to the line of sight. In order to be able to use polarimetric measurements the electron density $N_{e}$ has to be known. It is therefore also being identified thanks to interferometric measurements which give the density line integrals over each of the $N_{L}$ line of sights

$$
N_{e, o b s}^{i} \approx \int_{L^{i}} N e d l
$$

In NICE the electron density, assumed constant on magnetic surfaces, is represented as

$$
N_{e}\left(\psi_{\mathrm{N}}\right)=\lambda_{N e} \mathcal{C}\left(\psi_{\mathrm{N}}, \mathbf{u}_{\mathcal{C}}\right)
$$

where $\lambda_{N e}$ is a scaling parameter and function $\mathcal{C}$ is either decomposed in a basis of piecewise linear or cubic spline functions

$$
\mathcal{C}\left(x, \mathbf{u}_{\mathcal{C}}\right)=\sum_{i=1}^{N_{\mathcal{C}}} u_{\mathcal{C}, i} \phi_{i}(x)
$$


or given as a parametric function 33

$$
\mathcal{C}\left(x, \mathbf{u}_{\mathcal{C}}\right)=(M-m)(1-\alpha h z) E(z)+m
$$

where $E(z)=\frac{e^{-z}}{e^{-z}+e^{z}}, z=\frac{x-x_{0}}{h}$ and the vector of degrees of freedom is $\mathbf{u}_{\mathcal{C}}=\left(x_{0}, h, \alpha, m, M\right)$.

The interferometry observation operator $\mathbf{W}\left(\boldsymbol{\psi}, \mathbf{u}_{\mathcal{C}}\right)$ is a vector of size $N_{L}$ computed from the FEM discretiezation as

$$
\mathbf{W}\left(\boldsymbol{\psi}, \mathbf{u}_{\mathcal{C}}\right)_{i}=\int_{L^{i}} \lambda_{N_{e}} \mathcal{C}\left(\psi_{\mathrm{N}}\left(\psi_{h}\right), \mathbf{u}_{\mathcal{C}}\right) d l
$$

and for polarimetry we define $\mathbf{Z}\left(\boldsymbol{\psi}, \mathbf{u}_{\mathcal{C}}\right)$ in a similar way by

$$
\mathbf{Z}\left(\boldsymbol{\psi}, \mathbf{u}_{\mathcal{C}}\right)_{i}=\int_{L^{i}} c \lambda_{N_{e}} \mathcal{C}\left(\psi_{\mathrm{N}}\left(\psi_{h}\right), \mathbf{u}_{\mathcal{C}}\right) \mathbf{B}_{p}\left(\psi_{h}\right) \cdot \mathbf{t} d l
$$

Here again we have omitted normalization by the assumed observation error. Integrals along the lines of sight are approximated using the trapezoidal quadrature rule.

The vector of state variable is still $\mathbf{y}:=\boldsymbol{\psi}$ whereas the one of all control variables is now $\mathbf{u}=\left(\mathbf{u}_{\mathcal{A}}, \mathbf{u}_{\mathcal{B}}, \mathbf{u}_{\mathcal{C}}\right)$ and we can now define the cost function

$$
\begin{array}{r}
J(\mathbf{y}, \mathbf{u})=\frac{1}{2}\left\|\boldsymbol{H} \boldsymbol{\psi}+\boldsymbol{H}_{d} \boldsymbol{\psi}_{d}-\mathbf{m}\right\|^{2} \\
+\frac{1}{2}\left\|\mathbf{W}\left(\boldsymbol{\psi}, \mathbf{u}_{\mathcal{C}}\right)-\mathbf{m}_{N e}\right\|^{2}+\frac{1}{2}\left\|\mathbf{Z}\left(\boldsymbol{\psi}, \mathbf{u}_{\mathcal{C}}\right)-\mathbf{m}_{F a r}\right\|^{2} \\
+\frac{1}{2}\left\|\boldsymbol{R}_{\mathcal{A}} \mathbf{u}_{\mathcal{A}}\right\|^{2}+\frac{1}{2}\left\|\boldsymbol{R}_{\mathcal{B}} \mathbf{u}_{\mathcal{B}}\right\|^{2}+\frac{1}{2}\left\|\boldsymbol{R}_{\mathcal{C}} \mathbf{u}_{\mathcal{C}}\right\|^{2}
\end{array}
$$

which has to be minimized under the constraint of the equilibrium model (30).

In order to use the SQP algorithm we need to compute derivatives $\mathbf{W}_{\boldsymbol{\psi}}\left(\boldsymbol{\psi}, \mathbf{u}_{\mathcal{C}}\right)$ $\mathbf{W}_{\mathbf{u}_{\mathcal{C}}}\left(\boldsymbol{\psi}, \mathbf{u}_{\mathcal{C}}\right) \mathbf{Z}_{\boldsymbol{\psi}}\left(\boldsymbol{\psi}, \mathbf{u}_{\mathcal{C}}\right)$ and $\mathbf{Z}_{\mathbf{u}_{\mathcal{C}}}\left(\boldsymbol{\psi}, \mathbf{u}_{\mathcal{C}}\right)$. In NICE we compute the exact derivatives of the discrete operators. This is done on the fly in the same loop as the one used for the assembly of vectors $\mathbf{W}\left(\boldsymbol{\psi}, \mathbf{u}_{\mathcal{C}}\right)$ and $\mathbf{Z}\left(\boldsymbol{\psi}, \mathbf{u}_{\mathcal{C}}\right)$.

The computation of $\boldsymbol{\Delta} \mathbf{y}$ and $\boldsymbol{S}$ in (33) and (34) are unchanged. Concerning the gradients of the cost function we have

$$
\begin{aligned}
J_{\mathbf{y}}^{T}(\mathbf{y}, \mathbf{u}) & =\boldsymbol{H}^{T}\left(\boldsymbol{H} \boldsymbol{\psi}+\boldsymbol{H}_{d} \boldsymbol{\psi}_{d}-\mathbf{m}\right)+\mathbf{W}_{\boldsymbol{\psi}}\left(\boldsymbol{\psi}, \mathbf{u}_{\mathcal{C}}\right)^{T}\left(\mathbf{W}\left(\boldsymbol{\psi}, \mathbf{u}_{\mathcal{C}}\right)-\mathbf{m}_{N_{e}}\right) \\
& +\mathbf{Z}_{\boldsymbol{\psi}}\left(\boldsymbol{\psi}, \mathbf{u}_{\mathcal{C}}\right)^{T}\left(\mathbf{Z}\left(\boldsymbol{\psi}, \mathbf{u}_{\mathcal{C}}\right)-\mathbf{m}_{F a r}\right)
\end{aligned}
$$




$$
J_{\mathbf{u}}^{T}=\left[\begin{array}{c}
\boldsymbol{R}_{\mathcal{A}}^{T} \boldsymbol{R}_{\mathcal{A}} \mathbf{u}_{\mathcal{A}} \\
\boldsymbol{R}_{\mathcal{B}}^{T} \boldsymbol{R}_{\mathcal{B}} \mathbf{u}_{\mathcal{B}} \\
\boldsymbol{R}_{\mathcal{C}}^{T} \boldsymbol{R}_{\mathcal{C}} \mathbf{u}_{\mathcal{C}}+\mathbf{W}_{\mathbf{u}_{\mathcal{C}}}^{T}\left(\mathbf{W}-\mathbf{m}_{N_{e}}\right)+\mathbf{Z}_{\mathbf{u}_{\mathcal{C}}}^{T}\left(\mathbf{Z}-\mathbf{m}_{F a r}\right)
\end{array}\right]
$$

Concerning the second order derivative terms we avoid a complete computation by only considering terms involving products of first order derivatives and form the following quantities

$$
\begin{aligned}
& J_{\mathbf{y y}}=\boldsymbol{H}^{T} \boldsymbol{H}+\mathbf{W}_{\psi}^{T} \mathbf{W}_{\psi}+\mathbf{Z}_{\psi}^{T} \mathbf{Z}_{\psi} \\
& J_{\mathbf{u u}}=\left[\begin{array}{ccc}
\boldsymbol{R}_{\mathcal{A}}^{T} \boldsymbol{R}_{\mathcal{A}} & 0 & 0 \\
0 & \boldsymbol{R}_{\mathcal{B}}^{T} \boldsymbol{R}_{\mathcal{B}} & 0 \\
0 & 0 & \boldsymbol{R}_{\mathcal{C}}^{T} \boldsymbol{R}_{\mathcal{C}}+\mathbf{W}_{\mathbf{u}_{\mathcal{C}}}^{T} \mathbf{W}_{\mathbf{u}_{\mathcal{C}}}+\mathbf{Z}_{\mathbf{u}_{\mathcal{C}}}^{T} \mathbf{Z}_{\mathbf{u}_{\mathcal{C}}}
\end{array}\right] \\
& J_{\mathbf{y u}}=\left[\begin{array}{lll}
0 & 0 & \mathbf{W}_{\psi}^{T} \mathbf{W}_{\mathbf{u}_{\mathcal{C}}}+\mathbf{Z}_{\psi}^{T} \mathbf{Z}_{\mathbf{u}_{\mathcal{C}}}
\end{array}\right], \quad J_{\mathbf{u y}}=\left[\begin{array}{c}
0 \\
0 \\
\mathbf{W}_{\mathbf{u}_{\mathcal{C}}}^{T} \mathbf{W}_{\psi}+\mathbf{Z}_{\mathbf{u}_{\mathcal{C}}}^{T} \mathbf{Z}_{\psi}
\end{array}\right]
\end{aligned}
$$

Stokes model for polarimetry. The project of performing equilibrium reconstruction using interferometry and Stokes model for polarimetry is at the origin of the development of NICE. It was first presented in [4 where a Newton-Lagrange SQP algorithm is used in the ABB domain case for ITER. In this section we briefly restate the discretization method implemented for Stokes model and describe the more recent QSQP method used for the inverse equilibrium reconstruction problem. The method presented here was used in [34] with real measurements at JET.

Stokes model consists in a system of differential equations for the the Stokes vector $\mathbf{s}$ of dimension 3 to be solved along each line of sight. The system is linear in $\mathbf{s}$ but coefficients depend on the electron density and on the magnetic field. Each of the $N_{L}$ line of sight $L^{i}$ is discretized in $N^{i}$ points and a Crank-Nicolson scheme is implemented for the integration of the Stokes model. This can be written

$$
\boldsymbol{M}^{i}\left(\boldsymbol{\psi}, \mathbf{u}_{\mathcal{B}}, \mathbf{u}_{\mathcal{C}}\right) \mathbf{S}^{i}-\mathbf{S}_{0}^{i}=0
$$

where $\boldsymbol{M}^{i}$ is a $3 N^{i} \times 3 N^{i}$ band diagonal matrix, $\mathbf{S}^{i}=\left(\mathbf{s}^{i, 1}, \ldots, \mathbf{s}^{i, N^{i}}\right)$ is the vector of all Stokes vector states along the line of sight and $\mathbf{S}_{0}^{i}=\left(\mathbf{s}_{0}^{i}, 0, \ldots, 0\right)$ represents the initial conditions. 
The measured quantity can be assumed to be the Stokes vector at the end of the line of sight after integration through the plasma and the observation operator is given a matrix $\boldsymbol{E}^{i}$ of size $3 \times 3 N^{i}$ such that $\boldsymbol{E}^{i} \mathbf{S}^{i}=\mathbf{s}^{i, N^{i}}$.

Defining the block diagonal matrices

$$
\boldsymbol{M}=\operatorname{diag}\left(\boldsymbol{M}^{1}, \ldots, \boldsymbol{M}^{N_{L}}\right)
$$

and

$$
\boldsymbol{E}=\operatorname{diag}\left(\boldsymbol{E}^{1}, \ldots, \boldsymbol{E}^{N_{L}}\right),
$$

and collecting the Stokes vector states for all line of sights in a vector

$$
\mathbf{S}=\left(\mathbf{S}^{1}, \ldots, \mathbf{S}^{N_{L}}\right)
$$

one can define a direct model $\mathbf{e}(\mathbf{y}, \mathbf{u})=0$ for the state variable $\mathbf{y}=(\boldsymbol{\psi}, \mathbf{S})$ and control parameters $\mathbf{u}=\left(\mathbf{u}_{\mathcal{A}}, \mathbf{u}_{\mathcal{B}}, \mathbf{u}_{\mathcal{C}}\right)$ by

$$
\mathbf{e}(\mathbf{y}, \mathbf{u}):=\left[\begin{array}{c}
\boldsymbol{A} \boldsymbol{\psi}+\boldsymbol{A}_{d} \boldsymbol{\psi}_{d}-\mathbf{J}\left(\boldsymbol{\psi}, \lambda ; \mathbf{u}_{\mathcal{A}}, \mathbf{u}_{\mathcal{B}}\right)-\boldsymbol{L} \mathbf{u}_{I} \\
\boldsymbol{M}\left(\boldsymbol{\psi}, \mathbf{u}_{\mathcal{B}}, \mathbf{u}_{\mathcal{C}}\right) \mathbf{S}-\mathbf{S}_{0}
\end{array}\right]=0
$$

as well as the following cost function for equilibrium reconstruction using magnetics, interferometry and Stokes model polarimetry,

$$
\begin{gathered}
J(\mathbf{y}, \mathbf{u})=\frac{1}{2}\left\|\boldsymbol{H} \boldsymbol{\psi}+\boldsymbol{H}_{d} \boldsymbol{\psi}_{d}-\mathbf{m}\right\|^{2}+\frac{1}{2}\left\|\mathbf{W}\left(\boldsymbol{\psi}, \mathbf{u}_{\mathcal{C}}\right)-\mathbf{m}_{N e}\right\|^{2} \\
+\frac{1}{2}\left\|\mathbf{E S}-\mathbf{m}_{\mathbf{s}}\right\|^{2}+\frac{1}{2}\left\|\boldsymbol{R}_{\mathcal{A}} \mathbf{u}_{\mathcal{A}}\right\|^{2}+\frac{1}{2}\left\|\boldsymbol{R}_{\mathcal{B}} \mathbf{u}_{\mathcal{B}}\right\|^{2}+\frac{1}{2}\left\|\boldsymbol{R}_{\mathcal{C}} \mathbf{u}_{\mathcal{C}}\right\|^{2}
\end{gathered}
$$

which is minimized under the constraint of the model (equation 43).

The Jacobians needed in Eqn. (17) and (18) of the QSQP algorithm are

$$
\mathbf{e}_{\mathbf{y}}(\mathbf{y}, \mathbf{u})=\left[\begin{array}{cc}
\boldsymbol{A}-\mathbf{J}_{\boldsymbol{\psi}}\left(\boldsymbol{\psi}, \lambda ; \mathbf{u}_{\mathcal{A}}, \mathbf{u}_{\mathcal{B}}\right) & 0 \\
D_{\boldsymbol{\psi}}\left[\boldsymbol{M}\left(\boldsymbol{\psi}, \mathbf{u}_{\mathcal{B}}, \mathbf{u}_{\mathcal{C}}\right) \mathbf{S}\right] & \boldsymbol{M}\left(\boldsymbol{\psi}, \mathbf{u}_{\mathcal{B}}, \mathbf{u}_{\mathcal{C}}\right)
\end{array}\right]
$$

and

$\mathbf{e}_{\mathbf{u}}(\mathbf{y}, \mathbf{u})=\left[\begin{array}{ccc}-\mathbf{J}_{\mathbf{u}_{\mathcal{A}}}\left(\boldsymbol{\psi}, \lambda ; \mathbf{u}_{\mathcal{A}}, \mathbf{u}_{\mathcal{B}}\right) & -\mathbf{J}_{\mathbf{u}_{\mathcal{B}}}\left(\boldsymbol{\psi}, \lambda ; \mathbf{u}_{\mathcal{A}}, \mathbf{u}_{\mathcal{B}}\right) & 0 \\ 0 & D_{\mathbf{u}_{\mathcal{B}}}\left[\boldsymbol{M}\left(\boldsymbol{\psi}, \mathbf{u}_{\mathcal{B}}, \mathbf{u}_{\mathcal{C}}\right) \mathbf{S}\right] & D_{\mathbf{u}_{\mathcal{C}}}\left[\boldsymbol{M}\left(\boldsymbol{\psi}, \mathbf{u}_{\mathcal{B}}, \mathbf{u}_{\mathcal{C}}\right) \mathbf{S}\right]\end{array}\right]$

Concerning the gradients of the cost function we have

$$
J_{\mathbf{y}}^{T}(\mathbf{y}, \mathbf{u})=\left[\begin{array}{c}
\boldsymbol{H}^{T}\left(\boldsymbol{H} \boldsymbol{\psi}+\boldsymbol{H}_{d} \boldsymbol{\psi}_{d}-\mathbf{m}\right)+\mathbf{W}_{\boldsymbol{\psi}}\left(\boldsymbol{\psi}, \mathbf{u}_{\mathcal{C}}\right)^{T}\left(\mathbf{W}\left(\boldsymbol{\psi}, \mathbf{u}_{\mathcal{C}}\right)-\mathbf{m}_{N_{e}}\right) \\
\boldsymbol{E}^{T}\left(\boldsymbol{E} \mathbf{S}-\mathbf{m}_{\mathbf{s}}\right)
\end{array}\right]
$$




$$
J_{\mathbf{u}}^{T}=\left[\begin{array}{c}
\boldsymbol{R}_{\mathcal{A}}^{T} \boldsymbol{R}_{\mathcal{A}} \mathbf{u}_{\mathcal{A}} \\
\boldsymbol{R}_{\mathcal{B}}^{T} \boldsymbol{R}_{\mathcal{B}} \mathbf{u}_{\mathcal{B}} \\
\boldsymbol{R}_{\mathcal{C}}^{T} \boldsymbol{R}_{\mathcal{C}} \mathbf{u}_{\mathcal{C}}+\mathbf{W}_{\mathbf{u}_{\mathcal{C}}}^{T}\left(\mathbf{W}-\mathbf{m}_{N_{e}}\right)
\end{array}\right]
$$

Concerning the second order derivative terms we avoid a complete computation by only considering terms involving products of first order derivatives and form the following quantities

$$
\begin{aligned}
& J_{\mathbf{y y}}=\left[\begin{array}{cc}
\boldsymbol{H}^{T} \boldsymbol{H}+\mathbf{W}_{\boldsymbol{\psi}}^{T} \mathbf{W}_{\boldsymbol{\psi}} & 0 \\
0 & \boldsymbol{E}^{T} \boldsymbol{E}
\end{array}\right] \\
& J_{\mathbf{u u}}=\left[\begin{array}{ccc}
\boldsymbol{R}_{\mathcal{A}}^{T} \boldsymbol{R}_{\mathcal{A}} & 0 & 0 \\
0 & \boldsymbol{R}_{\mathcal{B}}^{T} \boldsymbol{R}_{\mathcal{B}} & 0 \\
0 & 0 & \boldsymbol{R}_{\mathcal{C}}^{T} \boldsymbol{R}_{\mathcal{C}}+\mathbf{W}_{\mathbf{u}_{\mathcal{C}}}^{T} \mathbf{W}_{\mathbf{u}_{\mathcal{C}}}
\end{array}\right] \\
& J_{\mathbf{y u}}=\left[\begin{array}{ccc}
0 & 0 & \mathbf{W}_{\boldsymbol{\psi}}^{T} \mathbf{W}_{\mathbf{u}_{\mathcal{C}}} \\
0 & 0 & 0
\end{array}\right], \quad J_{\mathbf{u y}}=\left[\begin{array}{cc}
0 & 0 \\
0 & 0 \\
\mathbf{W}_{\mathbf{u}_{\mathcal{C}}}^{T} \mathbf{W}_{\boldsymbol{\psi}} & 0
\end{array}\right]
\end{aligned}
$$

Finally let us mention that in [5] dependence on electron temperature is considered in the Stokes model and the numerical experiments are performed for the identification of the $T_{e}$ profile together with $p^{\prime}, f f^{\prime}$ and the $N_{e}$ profiles. As for other profiles the $T_{e}$ profile is decomposed in a function basis with control parameters $\mathbf{u}_{\mathcal{D}}$. With the notations of this Section this mainly consists in augmenting the control vector to $\mathbf{u}=\left(\mathbf{u}_{\mathcal{A}}, \mathbf{u}_{\mathcal{B}}, \mathbf{u}_{\mathcal{C}}, \mathbf{u}_{\mathcal{D}}\right)$ and adding a dependence of the Stokes model to $T_{e}$ that is to say using a matrix $\boldsymbol{M}\left(\boldsymbol{\psi}, \mathbf{u}_{\mathcal{B}}, \mathbf{u}_{\mathcal{C}}, \mathbf{u}_{\mathcal{D}}\right)$.

\subsubsection{Motional Start effect}

Motional Stark effect (MSE) measurements are angular measurements depending on the magnetic field inside the plasma domain. They are given at $N_{M S E}$ points $\mathbf{p}^{i}=\left(r^{i}, z^{i}\right)$ as

$$
m_{M S E}^{i}=\tan \gamma^{i}=\frac{a_{0}^{i} B_{z}\left(\mathbf{p}^{i}\right)+a_{1}^{i} B_{r}\left(\mathbf{p}^{i}\right)+a_{2}^{i} B_{\phi}\left(\mathbf{p}^{i}\right)}{a_{3}^{i} B_{z}\left(\mathbf{p}^{i}\right)+a_{4}^{i} B_{r}\left(\mathbf{p}^{i}\right)+a_{5}^{i} B_{\phi}\left(\mathbf{p}^{i}\right)}
$$

where the $a$ coefficients are given constants. In NICE the MSE observation operator $\mathbf{M}\left(\boldsymbol{\psi}, \boldsymbol{u}_{\mathcal{B}}\right)$ is the vector of components

$$
\mathbf{M}\left(\boldsymbol{\psi}, \boldsymbol{u}_{\mathcal{B}}\right)_{i}=\frac{a_{0}^{i} B_{z}\left(\psi_{h}\left(\mathbf{p}^{i}\right)\right)+a_{1}^{i} B_{r}\left(\psi_{h}\left(\mathbf{p}^{i}\right)\right)+a_{2}^{i} S_{f}\left(\psi_{\mathrm{N}}\left(\psi_{h}\left(\mathbf{p}^{i}\right)\right)\right) / r^{i}}{a_{3}^{i} B_{z}\left(\psi_{h}\left(\mathbf{p}^{i}\right)\right)+a_{4}^{i} B_{r}\left(\psi_{h}\left(\mathbf{p}^{i}\right)\right)+a_{5}^{i} S_{f}\left(\psi_{\mathrm{N}}\left(\psi_{h}\left(\mathbf{p}^{i}\right)\right)\right) / r^{i}}
$$


where we have noted $S_{f}\left(\psi_{\mathrm{N}}\right)=f(\psi)$ which depends on $\mathbf{u}_{\mathcal{B}}$ since it is obtained from integration of $\mathcal{B}$ as follows

$$
S_{f}\left(\psi_{\mathrm{N}}\right)=\left[\left(B_{0} r_{0}\right)^{2}-2\left(\psi_{b}-\psi_{a}\right) \lambda \mu_{0} r_{0} \int_{\psi_{\mathrm{N}}}^{1} \mathcal{B}(x) d x\right]^{1 / 2}
$$

$B_{0}$ is the vacuum toroidal field at $r=r_{0}$.

During assembling of vector $\mathbf{M}\left(\boldsymbol{\psi}, \boldsymbol{u}_{\mathcal{B}}\right)$ first order derivatives, $\mathbf{M}_{\boldsymbol{\psi}}\left(\boldsymbol{\psi}, \boldsymbol{u}_{\mathcal{B}}\right)$ and $\mathbf{M}_{\mathbf{u}_{\mathcal{B}}}\left(\boldsymbol{\psi}, \boldsymbol{u}_{\mathcal{B}}\right)$ are also computed. We can now formulate the cost function used for equilibrium reconstruction from magnetic and MSE measurement

$$
\begin{aligned}
J(\mathbf{y}, \mathbf{u}) & =\frac{1}{2}\left\|\boldsymbol{H} \boldsymbol{\psi}+\boldsymbol{H}_{d} \boldsymbol{\psi}_{d}-\mathbf{m}\right\|^{2}+\frac{1}{2}\left\|\mathbf{M}\left(\boldsymbol{\psi}, \mathbf{u}_{\mathcal{B}}\right)-\mathbf{m}_{M S E}\right\|^{2} \\
& +\frac{1}{2}\left\|\boldsymbol{R}_{\mathcal{A}} \mathbf{u}_{\mathcal{A}}\right\|^{2}+\frac{1}{2}\left\|\boldsymbol{R}_{\mathcal{B}} \mathbf{u}_{\mathcal{B}}\right\|^{2}
\end{aligned}
$$

which has to be minimized under the constraint of the equilibrium model (30).

The computation of $\Delta \mathbf{y}$ and $\boldsymbol{S}$ in $(33)$ and (34) are unchanged. Concerning the gradients of the cost function we have

$$
\begin{gathered}
J_{\mathbf{y}}^{T}(\mathbf{y}, \mathbf{u})=\boldsymbol{H}^{T}\left(\boldsymbol{H} \boldsymbol{\psi}+\boldsymbol{H}_{d} \boldsymbol{\psi}_{d}-\mathbf{m}\right)+\mathbf{M}_{\boldsymbol{\psi}}\left(\boldsymbol{\psi}, \mathbf{u}_{\mathcal{B}}\right)^{T}\left(\mathbf{M}\left(\boldsymbol{\psi}, \mathbf{u}_{\mathcal{B}}\right)-\mathbf{m}_{M S E}\right) \\
J_{\mathbf{u}}^{T}=\left[\begin{array}{c}
\boldsymbol{R}_{\mathcal{A}}^{T} \boldsymbol{R}_{\mathcal{A}} \mathbf{u}_{\mathcal{A}} \\
\boldsymbol{R}_{\mathcal{B}}^{T} \boldsymbol{R}_{\mathcal{B}} \mathbf{u}_{\mathcal{B}}+\mathbf{M}_{\mathbf{u}_{\mathcal{B}}}^{T}\left(\mathbf{M}-\mathbf{m}_{M S E}\right)
\end{array}\right]
\end{gathered}
$$

Very similar to what is done using interfero-polarimetry the following approximated second order derivative quantities are considered to form matrix $\boldsymbol{M}$ and vector $\mathbf{m}$ of the SQP algorithm

$$
\begin{gathered}
J_{\mathbf{y y}}=\boldsymbol{H}^{T} \boldsymbol{H}+\mathbf{M}_{\boldsymbol{\psi}}^{T} \mathbf{M}_{\boldsymbol{\psi}} \\
J_{\mathbf{u u}}=\left[\begin{array}{cc}
\boldsymbol{R}_{\mathcal{A}}^{T} \boldsymbol{R}_{\mathcal{A}} & 0 \\
0 & \boldsymbol{R}_{\mathcal{B}}^{T} \boldsymbol{R}_{\mathcal{B}}+\mathbf{M}_{\mathbf{u}_{\mathcal{B}}}^{T} \mathbf{M}_{\mathbf{u}_{\mathcal{B}}}
\end{array}\right] \\
J_{\mathbf{y u}}=\left[\begin{array}{lll}
0 & \mathbf{M}_{\psi}^{T} \mathbf{M}_{\mathbf{u}_{\mathcal{B}}}
\end{array}\right], \quad J_{\mathbf{u y}}=\left[\begin{array}{c}
0 \\
\mathbf{M}_{\mathbf{u}_{\mathcal{B}}}^{T} \mathbf{M}_{\boldsymbol{\psi}}
\end{array}\right]
\end{gathered}
$$




\subsubsection{Pressure}

The way pressure measurements can be obtained combining different diagnostics is out of the scope of this paper but when available they can be used for equilibrium reconstruction in NICE. Pressure measurements can be given as function of space, that is to say $N_{p}$ values $p^{i}$ given at points $\mathbf{p}^{i}=\left(r^{i}, z^{i}\right)$. They can also be given as a function $\psi_{\mathrm{N}}$ that is to say $N_{p}$ values $p^{i}$ given for $N^{p}$ values $\psi_{\mathrm{N}}^{i}$. In either case it leads to the construction of a pressure observation operator $\mathbf{P}\left(\boldsymbol{\psi}, \mathbf{u}_{\mathcal{A}}\right)$ with components

$$
\mathbf{P}\left(\boldsymbol{\psi}, \mathbf{u}_{\mathcal{A}}\right)_{i}=-\left(\psi_{b}-\psi_{a}\right) \frac{\lambda}{r_{0}} \int_{\psi_{\mathrm{N}}^{i}}^{1} \mathcal{A}(x) d x
$$

where $\psi_{\mathrm{N}}^{i}$ is either a fixed given quantity or computed as $\psi_{\mathrm{N}}\left(\psi_{h}\left(\mathbf{p}^{i}\right)\right)$ which leads to differences in the computation of derivatives $\mathbf{P}_{\boldsymbol{\psi}}\left(\boldsymbol{\psi}, \boldsymbol{u}_{\mathcal{A}}\right)$ and $\mathbf{P}_{\mathbf{u}_{\mathcal{A}}}\left(\boldsymbol{\psi}, \boldsymbol{u}_{\mathcal{A}}\right)$ done during the assembling. We can now formulate the cost function used for equilibrium reconstruction from magnetic and pressure measurements

$$
\begin{aligned}
J(\mathbf{y}, \mathbf{u}) & =\frac{1}{2}\left\|\boldsymbol{H} \boldsymbol{\psi}+\boldsymbol{H}_{d} \boldsymbol{\psi}_{d}-\mathbf{m}\right\|^{2}+\frac{1}{2}\left\|\mathbf{P}\left(\boldsymbol{\psi}, \mathbf{u}_{\mathcal{A}}\right)-\mathbf{m}_{p}\right\|^{2} \\
& +\frac{1}{2}\left\|\boldsymbol{R}_{\mathcal{A}} \mathbf{u}_{\mathcal{A}}\right\|^{2}+\frac{1}{2}\left\|\boldsymbol{R}_{\mathcal{B}} \mathbf{u}_{\mathcal{B}}\right\|^{2}
\end{aligned}
$$

which has to be minimized under the constraint of the equilibrium model (30).

The computation of $\Delta \mathbf{y}$ and $\boldsymbol{S}$ in (33) and (34) are unchanged. Concerning the gradients of the cost function we have

$$
\begin{gathered}
J_{\mathbf{y}}^{T}(\mathbf{y}, \mathbf{u})=\boldsymbol{H}^{T}\left(\boldsymbol{H} \boldsymbol{\psi}+\boldsymbol{H}_{d} \boldsymbol{\psi}_{d}-\mathbf{m}\right)+\mathbf{P}_{\boldsymbol{\psi}}\left(\boldsymbol{\psi}, \mathbf{u}_{\mathcal{A}}\right)^{T}\left(\mathbf{P}\left(\boldsymbol{\psi}, \mathbf{u}_{\mathcal{A}}\right)-\mathbf{m}_{p}\right) \\
J_{\mathbf{u}}^{T}=\left[\begin{array}{c}
\boldsymbol{R}_{\mathcal{A}}^{T} \boldsymbol{R}_{\mathcal{A}} \mathbf{u}_{\mathcal{A}}+\mathbf{P}_{\mathbf{u}_{\mathcal{A}}}^{T}\left(\mathbf{P}-\mathbf{m}_{p}\right) \\
\boldsymbol{R}_{\mathcal{B}}^{T} \boldsymbol{R}_{\mathcal{B}} \mathbf{u}_{\mathcal{B}}
\end{array}\right]
\end{gathered}
$$

Similar to what is done for interfero-polarimetry and MSE the following approximated second order derivative quantities are considered to form matrix $\boldsymbol{M}$ and vector $\mathbf{m}$ of the SQP algorithm

$$
\begin{gathered}
J_{\mathbf{y y}}=\boldsymbol{H}^{T} \boldsymbol{H}+\mathbf{P}_{\boldsymbol{\psi}}^{T} \mathbf{P}_{\boldsymbol{\psi}} \\
J_{\mathbf{u u}}=\left[\begin{array}{cc}
\boldsymbol{R}_{\mathcal{A}}^{T} \boldsymbol{R}_{\mathcal{A}}+\mathbf{P}_{\mathbf{u}_{\mathcal{A}}^{T}}^{\mathbf{P}_{\mathbf{u}_{\mathcal{A}}}} & 0 \\
0 & \boldsymbol{R}_{\mathcal{B}}^{T} \boldsymbol{R}_{\mathcal{B}}
\end{array}\right]
\end{gathered}
$$




$$
J_{\mathbf{y u}}=\left[\begin{array}{ll}
\mathbf{P}_{\psi}^{T} \mathbf{P}_{\mathbf{u}_{\mathcal{A}}} & 0
\end{array}\right], \quad J_{\mathbf{u y}}=\left[\begin{array}{c}
\mathbf{P}_{\mathbf{u}_{\mathcal{A}}^{T}}^{T} \mathbf{P}_{\psi} \\
0
\end{array}\right]
$$

\subsection{Outputs and uncertainty quantification}

4.3.1. Equilibrium outputs, flux surface averages and geometric coefficients

Numerous outputs can be extracted from the equilibrium poloidal flux map computed. These include purely geometric information on the plasma shape (plasma boundary, geometric axis, elongation ...), global parameters (such as total plasma current $I_{p}$, poloidal beta $\beta_{p}$, internal inductance $l i$, ...), 1D profiles of quantities constant on flux isolines in the plasma and 2D maps $\left(\psi\right.$ itself but also $\left.B_{r}, B_{z}, j_{p}, \ldots\right)$. All these outputs are standardized and follow the IMAS conventions. We are not going to detail all of them in this paper. Let us however give some details on the computation of some of the important $1 \mathrm{D}$ profiles in the plasma.

For $\psi_{\mathrm{N}} \in[0,1], S_{f}\left(\psi_{\mathrm{N}}\right)=f(\psi)$ is computed by integration of $\mathcal{B}$ as shown in (52). Let us define a discretization of the unit interval [0,1] by $S+1$ values $\psi_{\mathrm{N}}^{0}=0, \ldots, \psi_{\mathrm{N}}^{S}=1$. These points are taken as abscissa for all computed $1 \mathrm{D}$ profiles. For each $\psi_{\mathrm{N}}^{s}$ the contour line $\Gamma_{\psi_{\mathrm{N}}^{s}}$ is extracted from the finite element representation of the solution as a list of $N_{s}$ segments between $\mathbf{m}_{s, 1}^{l}=$ $\left(r_{s, 1}^{l}, z_{s, 1}^{l}\right)$ and $\mathbf{m}_{s, 2}^{l}=\left(r_{s, 2}^{l}, z_{s, 2}^{l}\right)$ with length $\left|L_{s}^{l}\right|$, for $l=1$ to $N_{s}$.

The toroidal flux coordinate is defined as $\rho\left(\psi_{\mathrm{N}}\right)=\sqrt{\phi\left(\psi_{\mathrm{N}}\right) / \pi B_{0}}$ where $\phi\left(\psi_{\mathrm{N}}\right)=\int_{\Omega_{\psi_{N}}} \frac{f\left(\psi_{h}(r, z)\right)}{r} d r d z$ and $\Omega_{\psi_{N}}$ is the domain bounded by the line of flux $\Gamma_{\psi_{\mathrm{N}}}$. This contour is not resolved by the mesh and the quantities $\phi_{s}$ and $\rho_{s}$ are computed from the discrete $\psi_{h}$ for all $\psi_{\mathrm{N}}^{s}$ using the same barycentric quadrature rule as for the integrated plasma current density $\mathbf{J}$ in Section 2.2.

$$
\phi_{s}=\sum_{T} \frac{S_{f}\left(\psi_{\mathrm{N}}\left(\mathbf{b}_{T}\left(\psi_{h}\right)\right)\right)}{r_{T}\left(\psi_{h}\right)}\left|T \cap \Omega_{\psi_{\mathrm{N}}^{s}}\right| .
$$

The profiles $\psi_{s}$ and $\rho_{s}$ being known one can compute $\left(\frac{\partial \psi}{\partial \rho}\right)_{s}=\psi_{s}^{\prime}$ using finite differences.

In the same way the volume profile is computed as

$$
V o l_{s}=2 \pi \sum_{T} r_{T}\left(\psi_{h}\right)\left|T \cap \Omega_{\psi_{\mathrm{N}}^{s}}\right|
$$

and $\left(\frac{\partial V o l}{\partial \rho}\right)_{s}=V o l_{s}^{\prime}$ using finite differences. 
Following [12] the average of a quantity $A$ over magnetic surfaces can be computed as

$$
\langle A\rangle_{s}=\left(\int_{\Gamma_{\psi_{\mathrm{N}}^{s}}} \frac{A r}{\left|\nabla \psi_{h}\right|} d l\right) /\left(\int_{\Gamma_{\psi_{\mathrm{N}}^{s}}} \frac{r}{\left|\nabla \psi_{h}\right|} d l\right) .
$$

A number of 1D profiles, also called geometric coefficients, are computed as such averages, e.g. $\left\langle 1 / r^{2}\right\rangle$ or $\left\langle|\nabla \rho|^{2} / r^{2}\right\rangle$. The integrals over flux contour lines involved are approximated as follows:

$$
\int_{\Gamma_{\psi_{\mathrm{N}}^{s}}} \frac{A r}{\left|\nabla \psi_{h}\right|} d l \approx \sum_{l=1}^{N_{s}} \frac{1}{2}\left(\frac{r_{s, 1}^{l} A\left(\mathbf{m}_{s, 1}^{l}\right)}{\mid \nabla \psi_{h\left|T_{s}^{l}\right|}}+\frac{r_{s, 2}^{l} A\left(\mathbf{m}_{s, 2}^{l}\right)}{\left|\nabla \psi_{h \mid T_{s}^{l}}\right|}\right)\left|L_{s}^{l}\right| .
$$

where $T_{s}^{l}$ is the triangle which is intersected by the segment between $\mathbf{m}_{s, 1}^{l}$ and $\mathbf{m}_{s, 2}^{l}$ and $\mathbf{m}_{s, \cdot}^{l}=\left(r_{s, \cdot}^{l}, z_{s, \cdot}^{l}\right) .\left|\nabla \psi_{h \mid T_{s}^{l}}\right|$ is constant in the triangle and computed from the 3 values at the nodes of $T_{s}^{l}$.

\subsubsection{Sensitivity method for error bars computation}

At convergence of the SQP iterations an optimal $\mathbf{u}$ is found and the constraint given by the model is satisfied. Hence $\mathbf{y}=\mathbf{y}(\mathbf{u})$ and one can define the reduced cost function $\hat{J}(\mathbf{u})=J(\mathbf{y}(\mathbf{u}), \mathbf{u})$. A good approximation to the Hessian of the reduced cost function is SQP algorithm matrix, $\hat{J}_{\mathbf{u u}} \approx \boldsymbol{M}$. This matrix would be the exact Hessian if we hadn't neglected some of the second order derivatives terms.

Its diagonal elements are the inverse of the squared a posteriori standard deviations on the components of $\mathbf{u}$ also called error bars. Moreover the error bar of any scalar quantity $g$ depending on $\mathbf{u}$ (such as the reconstructed functions $\mathcal{A}\left(\psi_{\mathrm{N}}\right)$ or $\mathcal{B}\left(\psi_{\mathrm{N}}\right)$ at a given $\psi_{\mathrm{N}}$ for example) is computed as

$$
\sigma_{g}^{2}=\nabla_{\mathbf{u}} g^{T} \boldsymbol{M}^{-1} \nabla_{\mathbf{u}} g
$$

This enables the computation of the error bar on any output quantity at low numerical cost. If $g(\mathbf{u})$ is in the form $g(\mathbf{u})=G(\mathbf{y}(\mathbf{u}), \mathbf{u})$ we use

$$
\nabla_{\mathbf{u}} g=\mathbf{y}_{\mathbf{u}}^{T} \nabla_{\mathbf{y}} G+\nabla_{\mathbf{u}} G
$$

where the Jacobian $\mathbf{y}_{\mathbf{u}}=\boldsymbol{S}$ is already known from SQP algorithm. 


\subsection{Final remarks on equilibrium reconstruction}

The equilibrium reconstruction problem can be formulated on the $\mathrm{ABB}$ domain. With this formulation magnetic Bprobes and flux loops measurements are directly used without having to perform a first interpolation step on the measurement contour as with the formulation on a bounded domain. However there are two drawbacks to this method. First of all it is computationally more demanding since the mesh is necessarily much larger, and secondly it does not work well for tokamaks with ferromagnetic structures since the modelization of the magnetic permeability is certainly not very precise. Nevertheless the method works well for iron-free tokamaks and a numerical example is provided in Section 5.3. This method is closer to the algorithms implemented in codes like EFIT [35, EQUAL 36, CLISTE [37] or LIUQE [38], which however do not make use of derivative with respect to the state variable as is done here in the QSQP algorithm.

The cost function to be minimized is very similar to 29

$$
\begin{aligned}
J(\boldsymbol{\psi}, \mathbf{u})= & \frac{1}{2}\left\|\boldsymbol{H}_{B} \boldsymbol{\psi}-\mathbf{m}_{B}\right\|^{2}+\frac{1}{2}\left\|\boldsymbol{H}_{F} \boldsymbol{\psi}-\mathbf{m}_{F}\right\|^{2} \\
& +\frac{1}{2}\left\|\boldsymbol{R}_{\mathcal{A}} \mathbf{u}_{\mathcal{A}}\right\|^{2}+\frac{1}{2}\left\|\boldsymbol{R}_{\mathcal{B}} \mathbf{u}_{\mathcal{B}}\right\|^{2}
\end{aligned}
$$

Here $\mathbf{m}_{B}$ (resp. $\mathbf{m}_{F}$ ) are the Bprobes measurements (resp. the flux loops measurements) and $\boldsymbol{H}_{B}$ (resp. $\boldsymbol{H}_{F}$ ) the associated linear observation operators mapping the FEM representation of the poloidal flux to the measurements.

For an iron-free tokamak the model equation constraint reads

$$
(\boldsymbol{A}+\boldsymbol{C}) \boldsymbol{\psi}-\mathbf{J}\left(\boldsymbol{\psi}, \lambda, \mathbf{u}_{\mathcal{A}}, \mathbf{u}_{\mathcal{B}}\right)-\boldsymbol{L} \mathbf{u}_{I}=0
$$

The QSQP algorithm follows as in the preceding sections.

Clearly the different measurements of the previous sections (magnetics, interferometry, polarimetry, MSE, pressure) can be used all together. In NICE this is controled by code parameters and the user can decide to use any combination of measurements. Finally one can also perform a magnetics only reconstruction and then use the obtained flux map to reconstruct in a second step the electron density profile using interferometry measurements only. 


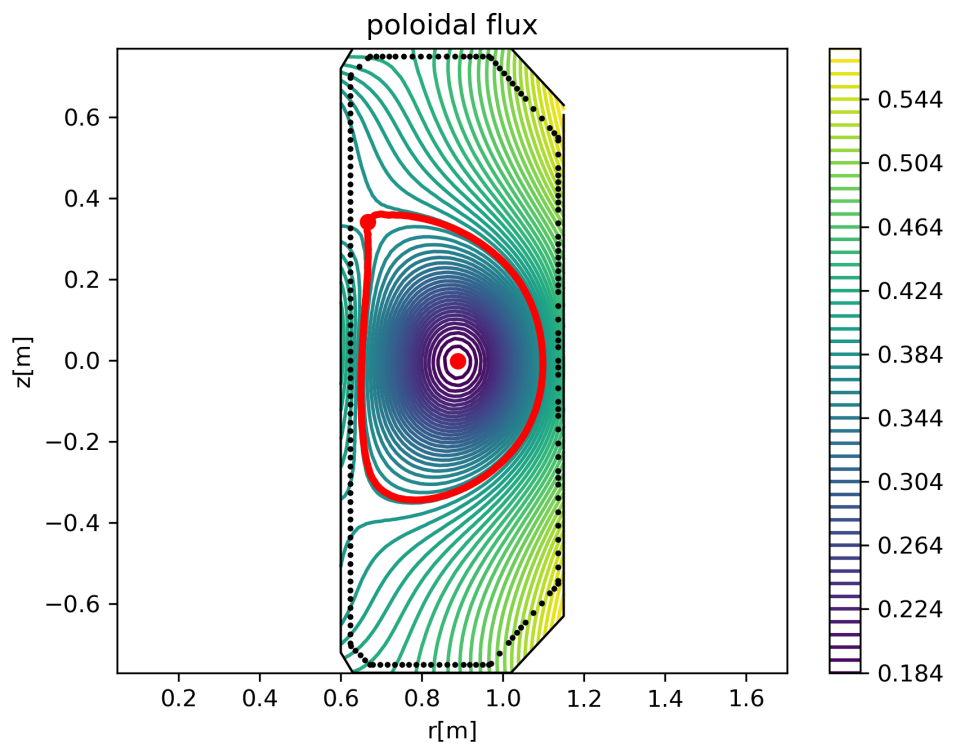

Figure 9: Poloidal flux map for a TCV-like equilibrium reconstruction with magnetic measurements. The plasma boundary is shown in red. The limiter contour is the doted line.

\subsection{Numerical examples}

$T C V$-like equilibrium reconstruction with magnetics only. This first example shows results from a TCV-like equilibrium reconstrucion using magnetics only. Figure 9 shows the computation domain and the obtained flux map. Cauchy measurements on the domain boundary are computed with the first mode of NICE described in Section 3 using toroidal harmonics of order 5, then the QSQP algorithm of section 4.1 is run. Functions $\mathcal{A}$ and $\mathcal{B}$ are decomposed in a basis of 6 cubic splines each. The regularization parameters are $\varepsilon_{\mathcal{A}}=\varepsilon_{\mathcal{B}}=0.1$. The relative residual, relresid $=\frac{\left\|(\mathbf{y}, \mathbf{u})^{n+1}-(\mathbf{y}, \mathbf{u})^{n}\right\|}{\left\|(\mathbf{y}, \mathbf{u})^{n}\right\|}$, rapidly converges to the tolerance value set to $10^{-10}$. Figure 10 shows the identified $p^{\prime}$ and $f f^{\prime}$ profiles as well as the obtained averaged current density and safety factor. Computed error bars on these profiles are also shown.

WEST-like equilibrium reconstruction with magnetics and interfero-polarimetry. This second example shows results for a WEST-like equilibrium reconstrucion using magnetics and interfero-polarimetry measurements. Figure 11 shows 

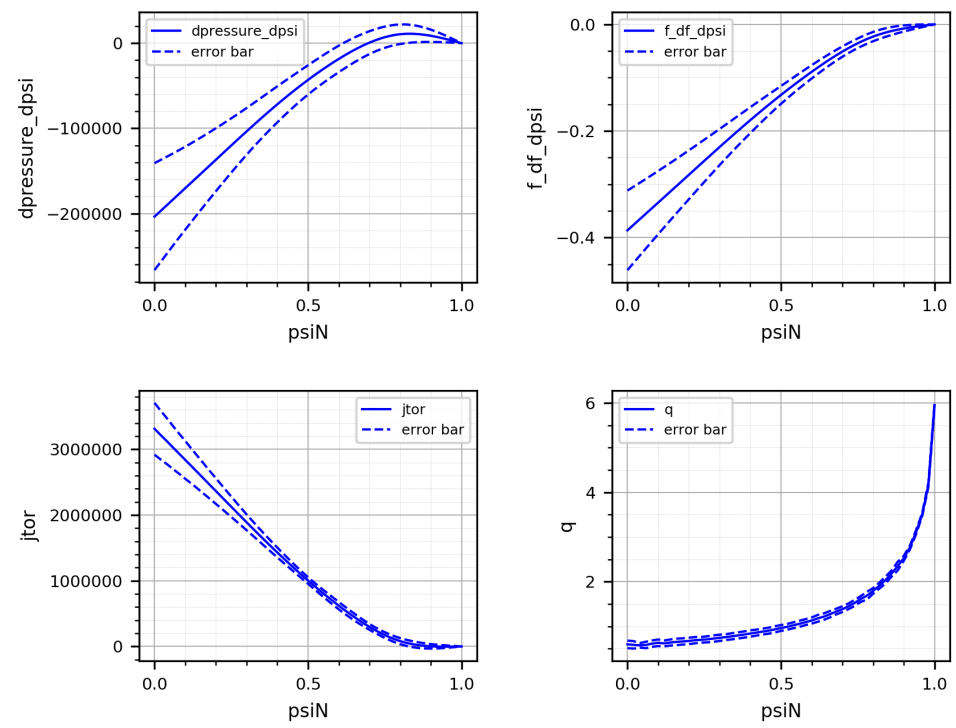

Figure 10: TCV-like equilibrium reconstruction with magnetics. Reconstructed $p^{\prime}, f f^{\prime}$, $j_{\text {tor }}=\left(p^{\prime}+<1 / r^{2}>\left(1 / \mu_{0}\right) f f^{\prime}\right) /<1 / r>$ and safety factor $q$ profiles, and associated computed error bars. 


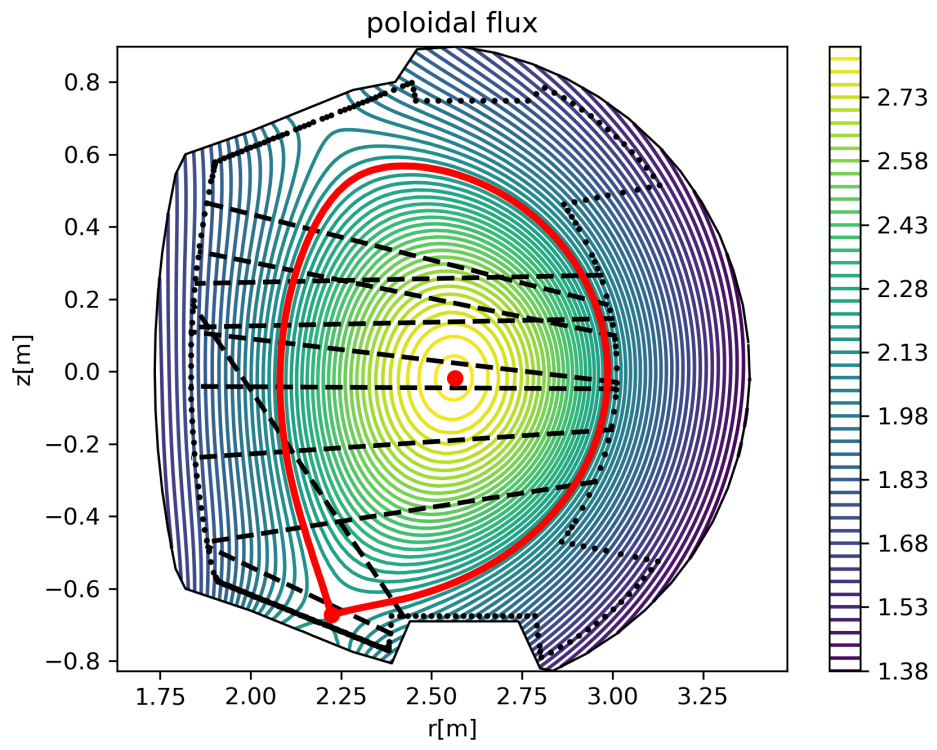

Figure 11: Poloidal flux map for a WEST-like equilibrium reconstruction with magnetics and interfero-polarimetry. The plasma boundary is shown in red. The limiter contour is the doted line and the dashed lines are the interfero-polarimetry chords

the computation domain and the obtained flux map. Cauchy measurements on the domain boundary are computed with the first mode of NICE described in Section 3 using external harmonics of order 7 and inner of order 4, then the QSQP algorithm of section 4.2.1 is run. Functions $\mathcal{A}$ and $\mathcal{B}$ are decomposed in a basis of 7 cubic splines each whereas $\mathcal{C}$ uses 8 . The regularization parameters are $\varepsilon_{\mathcal{A}}=\varepsilon_{\mathcal{B}}=0.1$. The regularization parameter $\varepsilon_{\mathcal{C}}$ varies radially from 1 on the magnetic axis to $10^{-2}$ at the plasma boundary. Values of $\mathcal{A}$ and $\mathcal{B}$ at the plasma boundary are forced to 0 with large penalization parameters whereas $\mathcal{C}$ is left free. The relative residual rapidly converges to the tolerance value set to $10^{-10}$. Figure 12 shows the identified $p^{\prime}$ and $f f^{\prime}$ profiles as well as the obtained averaged current density and safety factor. Computed error bars on these profiles are also shown. Figure 13 shows the identified electron density profile with error bars as well as the interferometry and polarimetry data. Because of invalid measurements 8 chords out of 10 are used for interferometry while 7 are used for polarimetry. 

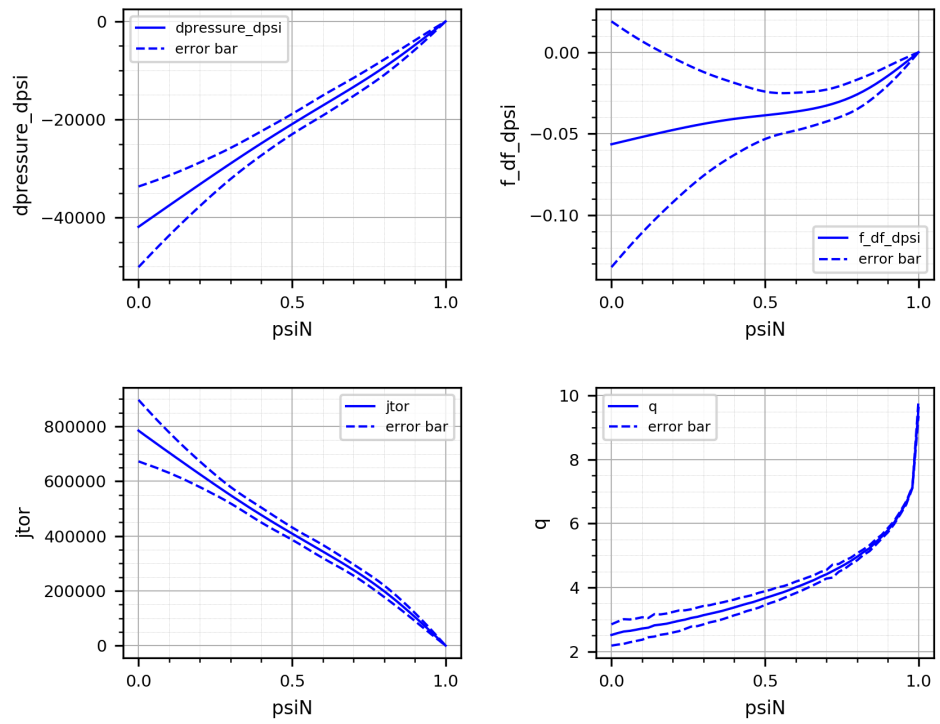

Figure 12: WEST-like equilibrium reconstruction with magnetics and interferopolarimetry. Reconstructed $p^{\prime}, f f^{\prime}, j_{\text {tor }}=\left(p^{\prime}+<1 / r^{2}>\left(1 / \mu_{0}\right) f f^{\prime}\right) /<1 / r>$ and safety factor $q$ profiles, and associated computed error bars. 

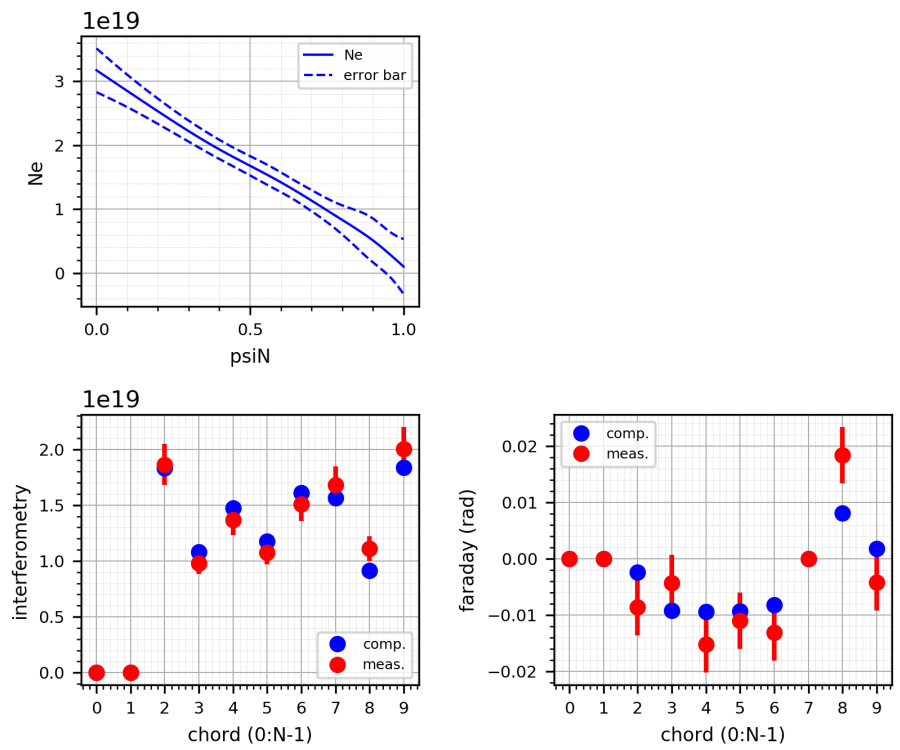

Figure 13: WEST-like equilibrium reconstruction with magnetics and interferopolarimetry. Reconstructed electron density $N_{e}$ and associated computed error bars. Computed and measured interferometry and Faraday angle data. 
JET-like equilibrium reconstruction with magnetics and pressure. This third example shows results for a JET-like equilibrium reconstrucion using magnetics and pressure measurements. Pressure measurements are calculated from High Resolution Thomson Scattering diagnostic with the assumption $n_{i}=n_{e}$ and $T_{i}=T_{e}$, and data mapped onto flux surface coordinates. Figure 14 shows the computation domain and the obtained flux map. Cauchy measurements on the domain boundary are computed with the first mode of NICE described in Section 3 using external harmonics of order 5 and inner of order 3, then the QSQP algorithm of section 4.2.3 is run. Function $\mathcal{A}$ is decomposed in a basis of 14 cubic splines and the regularization parameter $\varepsilon_{\mathcal{A}}$ varies radially from $10^{-1}$ on the magnetic axis to $10^{-3}$ near the plasma boundary where the function is left free. Function $\mathcal{B}$ is decomposed in a basis of 12 cubic splines and the regularization parameter is $\varepsilon_{\mathcal{A}}=10^{-1}$ and $\mathcal{B}(1)$ is forced to 0 with a large penalization parameter. The relative residual rapidly converges to the tolerance value set to $10^{-10}$. Figure 15 shows the identified $p^{\prime}$ and $f f^{\prime}$ profiles as well as the obtained averaged current density and safety factor. Computed error bars on these profiles are also shown. Figure 16 shows the identified pressure profile with error bars as well as the pressure data.

\section{NICE direct and inverse equilibrium computation modes}

\subsection{Static equilibrium computation}

Two types of direct static equilibrium computation can be performed with NICE, $I_{p}$-free (in which the total plasma current is not imposed) or $I_{p}$-fixed computations (in which the total plasma current is imposed to a given value). Although it is possible to perform this type of computation in the bounded domain case it is most usually performed in the ABB domain case where all poloidal field coils are included. In both types of computation the currents in these coils are given, that is $\mathbf{u}:=\mathbf{u}_{I}$ the control variable for the inverse problem to come, is a given fixed parameter.

In $I_{p}$-free computations the current density functions $p^{\prime}$ and $f f^{\prime}$ are given, that is $\lambda, \mathbf{u}_{\mathcal{A}}$ and $\mathbf{u}_{\mathcal{B}}$ are given and fixed. The state variable is $\mathbf{y}:=\boldsymbol{\psi}$ and the model equation is

$$
\mathbf{e}(\mathbf{y}, \mathbf{u}):=(\boldsymbol{A}+\boldsymbol{C}) \boldsymbol{\psi}+\mathbf{A}_{\mu}(\boldsymbol{\psi})-\mathbf{J}\left(\boldsymbol{\psi}, \lambda ; \mathbf{u}_{\mathcal{A}}, \mathbf{u}_{\mathcal{B}}\right)-\boldsymbol{L} \mathbf{u}_{I}=0 .
$$

In $I_{p}$-fixed computations the current density functions are given up to the 


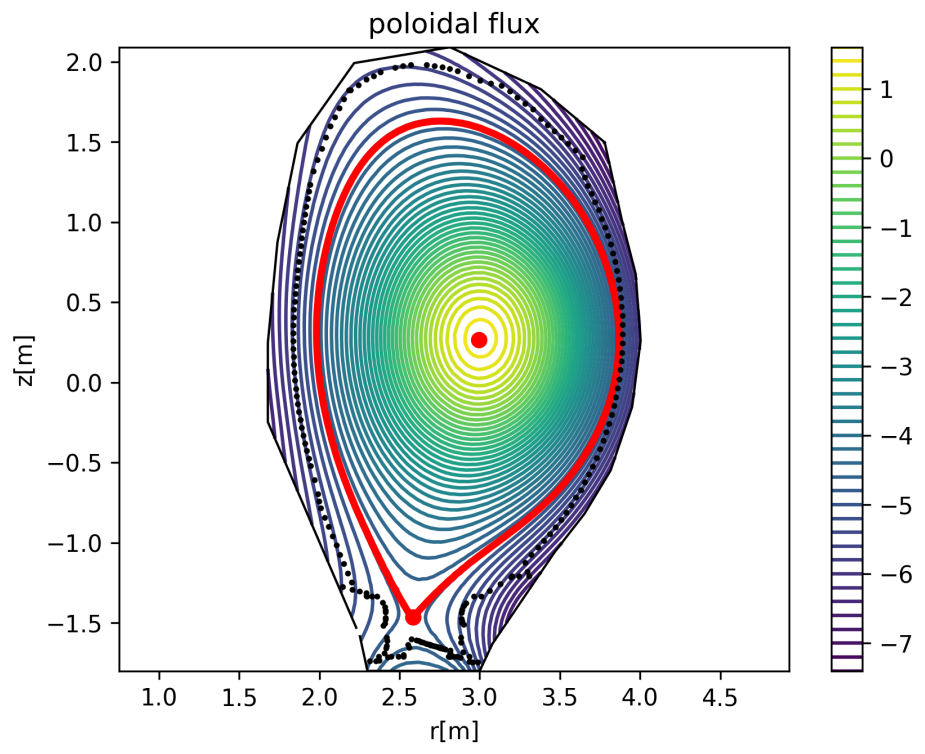

Figure 14: Poloidal flux map for a JET-like equilibrium reconstruction with magnetics and pressure measurements. The plasma boundary is shown in red. The limiter contour is the doted line. 

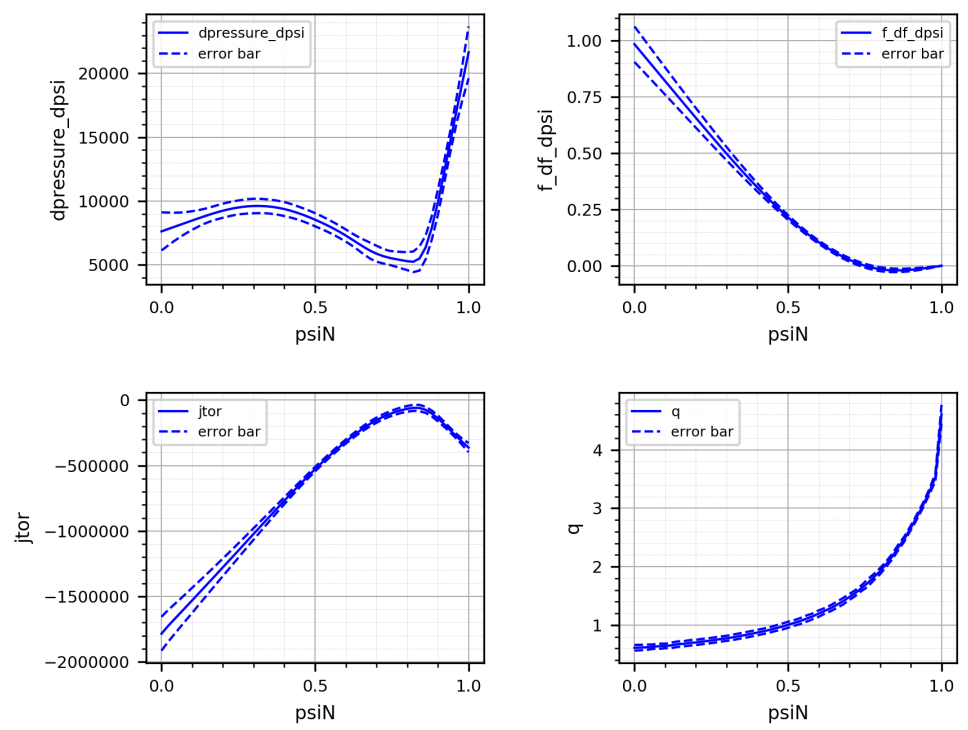

Figure 15: JET-like equilibrium reconstruction with magnetics and pressure. Reconstructed $p^{\prime}, f f^{\prime}, j_{\text {tor }}=\left(p^{\prime}+<1 / r^{2}>\left(1 / \mu_{0}\right) f f^{\prime}\right) /<1 / r>$ and safety factor $q$ profiles, and associated computed error bars. 

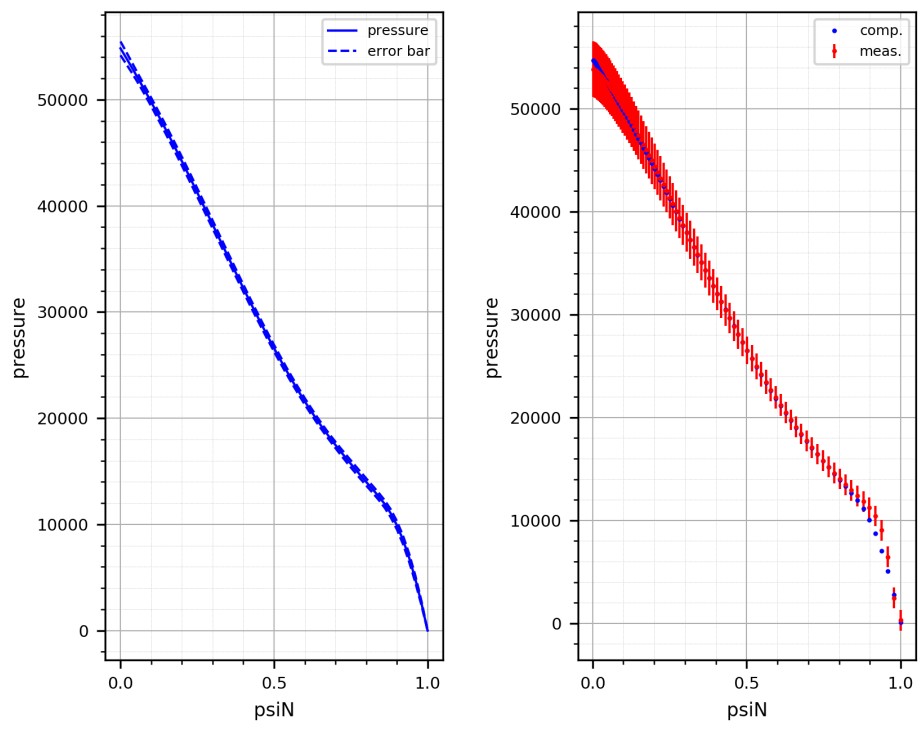

Figure 16: JET-like equilibrium reconstruction with magnetics and pressure. Reconstructed pressure $p$ and associated computed error bars. Computed and measured pressure data. 
scaling factor $\lambda$. The state variable is augmented with $\lambda, \mathbf{y}:=(\boldsymbol{\psi}, \lambda)$ and the model equation augmented with an equation imposing a given $I_{p}$ value

$$
\mathbf{e}(\mathbf{y}, \mathbf{u}):=\left[\begin{array}{c}
(\boldsymbol{A}+\boldsymbol{C}) \boldsymbol{\psi}+\mathbf{A}_{\mu}(\boldsymbol{\psi})-\mathbf{J}\left(\boldsymbol{\psi}, \lambda ; \mathbf{u}_{\mathcal{A}}, \mathbf{u}_{\mathcal{B}}\right)-\boldsymbol{L} \mathbf{u}_{I} \\
I_{p}-\lambda J_{p}\left(\boldsymbol{\psi}, \mathbf{u}_{\mathcal{A}}, \mathbf{u}_{\mathcal{B}}\right)
\end{array}\right]=0 .
$$

The direct static $I_{p}$-free or $I_{p}$-fixed computation consists in finding $\mathbf{y}$ such that $\mathbf{e}(\mathbf{y}, \mathbf{u})=0$ using Newton's method.

The associated inverse problem is to find the currents in the coils, hence $\mathbf{u}$ such that the plasma has a desired shape and position. A number $N_{p}+1$ of points $\mathbf{x}_{i}=\left(r_{i}, z_{i}\right)$ defining the desired plasma boundary are provided. They are either given from another simulation or provided from the parametric representation taken from [21, Chap. 4, p84]. A typical cost function is

$$
J(\mathbf{y}, \mathbf{u})=\frac{1}{2}\|\boldsymbol{K} \boldsymbol{\psi}\|^{2}+\frac{1}{2}\left\|\boldsymbol{R} \mathbf{u}_{I}\right\|^{2}
$$

where $\|\boldsymbol{K} \boldsymbol{\psi}\|^{2}=\sum_{i=1}^{N_{p}}\left(\psi_{h}\left(\mathbf{x}_{i}\right)-\psi_{h}\left(\mathbf{x}_{0}\right)\right)^{2}$ is the misfit term imposing an isoflux line to go through the given desired points and the second term is a penalization term in which the diagonal matrix $\boldsymbol{R}$ holds weights $R_{i i}=1 / \sigma_{i}^{2}$.

As for the equilibrium reconstruction problem the inverse problem

$$
\min _{\mathbf{y}, \mathbf{u}} J(\mathbf{y}, \mathbf{u}) \text { such that } \mathbf{e}(\mathbf{y}, \mathbf{u})=0
$$

is solved thanks to the QSQP method. The needed ingredients are

$$
\begin{gathered}
\mathbf{e}_{\mathbf{y}}(\mathbf{y}, \mathbf{u})=\boldsymbol{A}+\boldsymbol{C}+\mathbf{A}_{\mu, \boldsymbol{\psi}}(\boldsymbol{\psi})-\mathbf{J}_{\boldsymbol{\psi}}\left(\boldsymbol{\psi}, \lambda ; \mathbf{u}_{\mathcal{A}}, \mathbf{u}_{\mathcal{B}}\right), \quad \mathbf{e}_{\mathbf{u}}(\mathbf{y}, \mathbf{u})=-\boldsymbol{L} \\
J_{\mathbf{y}}^{T}(\mathbf{y}, \mathbf{u})=\boldsymbol{K}^{T} \boldsymbol{K} \boldsymbol{\psi}, \quad J_{\mathbf{u}}^{T}(\mathbf{y}, \mathbf{u})=\boldsymbol{R}^{T} \boldsymbol{R} \mathbf{u}_{I} \\
J_{\mathbf{y y}}(\mathbf{y}, \mathbf{u})=\boldsymbol{K}^{T} \boldsymbol{K}, \quad J_{\mathbf{u u}}(\mathbf{y}, \mathbf{u})=\boldsymbol{R}^{T} \boldsymbol{R}, \quad J_{\mathbf{y u}}=J_{\mathbf{u y}}=0
\end{gathered}
$$

for the $I_{p}$-free case and

$$
\mathbf{e}_{\mathbf{y}}(\mathbf{y}, \mathbf{u})=\left[\begin{array}{cc}
\boldsymbol{A}+\boldsymbol{C}+\mathbf{A}_{\mu, \boldsymbol{\psi}}(\boldsymbol{\psi})-\mathbf{J}_{\boldsymbol{\psi}}\left(\boldsymbol{\psi}, \lambda ; \mathbf{u}_{\mathcal{A}}, \mathbf{u}_{\mathcal{B}}\right) & -\mathbf{J}_{\lambda}\left(\boldsymbol{\psi}, \lambda ; \mathbf{u}_{\mathcal{A}}, \mathbf{u}_{\mathcal{B}}\right) \\
-\lambda J_{p, \boldsymbol{\psi}}\left(\boldsymbol{\psi}, \mathbf{u}_{\mathcal{A}}, \mathbf{u}_{\mathcal{B}}\right) & -J_{p}\left(\boldsymbol{\psi}, \mathbf{u}_{\mathcal{A}}, \mathbf{u}_{\mathcal{B}}\right)
\end{array}\right]
$$




$$
\begin{gathered}
\mathbf{e}_{\mathbf{u}}(\mathbf{y}, \mathbf{u})=\left[\begin{array}{c}
-\boldsymbol{L} \\
0
\end{array}\right] \\
J_{\mathbf{y}}^{T}(\mathbf{y}, \mathbf{u})=\left[\begin{array}{c}
\boldsymbol{K}^{T} \boldsymbol{K} \boldsymbol{\psi} \\
0
\end{array}\right], \quad J_{\mathbf{u}}^{T}(\mathbf{y}, \mathbf{u})=\boldsymbol{R}^{T} \boldsymbol{R} \mathbf{u}_{I} \\
J_{\mathbf{y y}}(\mathbf{y}, \mathbf{u})=\left[\begin{array}{cc}
\boldsymbol{K}^{T} \boldsymbol{K} & 0 \\
0 & 0
\end{array}\right], \quad J_{\mathbf{u u}}(\mathbf{y}, \mathbf{u})=\boldsymbol{R}^{T} \boldsymbol{R}, \quad J_{\mathbf{y u}}=J_{\mathbf{u y}}=0
\end{gathered}
$$

for the $I_{p}$-fixed case.

\subsection{Quasi-static equilibrium evolution computation}

In order to compute the quasi-static equilibrium evolution on time interval $[0, T]$ an implicit Euler scheme with time step $\Delta t$ is used starting from an initial condition $\mathbf{y}^{0}$. The computation of the equilibrium at $t^{k}$ from the one at $t^{k-1}$ is done by solving the following problem:

$$
\text { find } \mathbf{y}^{k} \quad \text { such that } \mathbf{e}^{k}\left(\mathbf{y}^{k}, \mathbf{y}^{k-1}, \mathbf{u}_{V}\right)=0
$$

where

$$
\begin{aligned}
\mathbf{e}^{k}\left(\mathbf{y}^{k}, \mathbf{y}^{k-1}, \mathbf{u}_{V}\right):= & (\boldsymbol{A}+\boldsymbol{C}) \boldsymbol{\psi}^{k}+\mathbf{A}_{\mu}\left(\boldsymbol{\psi}^{k}\right)-\mathbf{J}\left(\boldsymbol{\psi}^{k}, \lambda^{k}, \mathbf{u}_{\mathcal{A}}^{k}, \mathbf{u}_{\mathcal{B}}^{k}\right) \\
& -\boldsymbol{E}\left(\boldsymbol{\psi}^{k}-\boldsymbol{\psi}^{k-1}\right)-\boldsymbol{G}^{k} \mathbf{u}_{V}
\end{aligned}
$$

with $\mathbf{y}^{k}:=\boldsymbol{\psi}^{k}$ in the $I_{p}$-free case.

Matrices $\boldsymbol{E}$ and $\boldsymbol{G}^{k}$ are defined as

$$
\boldsymbol{E}:=\frac{1}{\Delta t}\left(\boldsymbol{J}_{p s}+\boldsymbol{L} \boldsymbol{R} \boldsymbol{L}^{T}\right)
$$

and

$$
\boldsymbol{G}^{k}:=\boldsymbol{L} \boldsymbol{S} \boldsymbol{B}^{k} .
$$

For the the $I_{p}$-fixed case we have

$$
\mathbf{e}^{k}\left(\mathbf{y}^{k}, \mathbf{y}^{k-1}, \mathbf{u}_{V}\right):=\left[\begin{array}{c}
(\boldsymbol{A}+\boldsymbol{C}) \boldsymbol{\psi}^{k}+\mathbf{A}_{\mu}\left(\boldsymbol{\psi}^{k}\right)-\mathbf{J}\left(\boldsymbol{\psi}^{k}, \lambda^{k}, \mathbf{u}_{\mathcal{A}}^{k}, \mathbf{u}_{\mathcal{B}}^{k}\right) \ldots \\
\cdots-\boldsymbol{E}\left(\boldsymbol{\psi}^{k}-\boldsymbol{\psi}^{k-1}\right)-\boldsymbol{G}^{k} \mathbf{u}_{V} \\
I_{p}^{k}-\lambda^{k} J_{p}\left(\boldsymbol{\psi}^{k}, \mathbf{u}_{\mathcal{A}}^{k}, \mathbf{u}_{\mathcal{B}}^{k}\right)
\end{array}\right]
$$

with $\mathbf{y}^{k}:=\left(\boldsymbol{\psi}^{k} ; \lambda^{k}\right)$. 
Similar to Section 5.1, in $I_{p}$-free computations the current density functions $p^{\prime}$ and $f f^{\prime}$ are given that is $\lambda^{k}, \mathbf{u}_{\mathcal{A}}^{k}$ and $\mathbf{u}_{\mathcal{B}}^{k}$ are given at each time step. In $I_{p}$-fixed computations the current density functions are given up to the scaling factor $\lambda$ which is computed at each time given a prescribed value of the plasma current $I_{p}^{k}$.

The voltage $v_{i}(t)$ of each of the $N_{v}$ power supplies is a given function of time decomposed in a basis of $N_{b v}$ piecewise linear or spline functions

$$
v_{i}(t)=\sum_{j=1}^{N_{b v}} u_{i, j} \phi_{j}(t)
$$

Noting $\mathbf{u}_{V}$ the vector of size $N_{v} N_{b v}$ of all decomposition coefficients $u_{i, j}$ for all power supllies, the $N_{v} \times N_{v} N_{b v}$ matrix $\boldsymbol{B}(t)$ is such that $\boldsymbol{B}(t) \mathbf{u}_{V}=\mathbf{v}(t)$ the vector of all voltages.

As for the static case inverse problems can be defined for the quasi-static evolution case. It consists in finding the voltages, hence controling $\mathbf{u}=$ $\mathbf{u}_{V}$, such that the plasma shape follows a prescribed evolution. In order to stick with the notations of this document one can define a state variable concatenating the states at each time step $\mathbf{y}:=\left(\mathbf{y}^{1}, \ldots \mathbf{y}^{N_{T}}\right)$ and a model

$$
\boldsymbol{e}(\mathbf{y}, \mathbf{u}):=\left[\begin{array}{c}
\mathbf{e}^{1}\left(\mathbf{y}^{1}, \mathbf{y}^{0}, \mathbf{u}_{V}\right) \\
\cdots \\
\mathbf{e}^{N_{T}}\left(\mathbf{y}^{N_{T}}, \mathbf{y}^{N_{T}-1}, \mathbf{u}_{V}\right)
\end{array}\right]
$$

The simplest cost function which can be defined is the following

$$
J(\mathbf{y}, \mathbf{u})=\frac{1}{2} \sum_{k=1}^{N_{T}} \sum_{i=1}^{N_{d}} w_{k}\left(\psi_{h}^{k}\left(\mathbf{x}_{i}^{k}\right)-\psi_{h}^{k}\left(\mathbf{x}_{0}^{k}\right)\right)^{2}+\frac{1}{2} w \sum_{k=1}^{N_{T}} \sum_{i=1}^{N_{v}}\left(\left[\boldsymbol{B}^{k} \mathbf{u}_{V}\right]_{i}\right)^{2}
$$

with a misfit term to a desired plasma boundary at each time step and a penalization term on voltages. This can be rewritten

$$
J(\mathbf{y}, \mathbf{u})=\frac{1}{2}\|\boldsymbol{K} \boldsymbol{\psi}\|^{2}+\frac{1}{2}\left\|\boldsymbol{R} \mathbf{u}_{V}\right\|^{2}
$$

and the QSQP algorithm to solve the PDE-constrained optimization problem follows. Other types of penalization terms are also implemented in NICE: penalization on the maximum and minimum voltage values and penalization on induced currents in passive strutures. we refer to [6] for a study of this type of inverse problems for the design of tokamak scenarios. 


\subsection{Numerical examples}

JT60-SA equilibrium reconstruction in ABB domain with synthetic magnetics generated from a static inverse. In this first example we perform an equilibrium reconstruction with synthetic magnetic data for JT60-SA tokamak in $\mathrm{ABB}$ domain. In a first step, the Ip-fixed static inverse mode of NICE is run to compute the currents in the PF coils giving a desired plasma boundary shape provided as a list of input points. The 12 reference currents in (73) are $\mathbf{u}_{I_{0}}=0$ and the penalization weights are chosen as $\sigma_{i}=10^{8}$. A parametric representation for $\mathcal{A}$ and $\mathcal{B}$ is given. The relative residual rapidly converges to $10^{-10}$.

This provides a reference equilibrium with known $p^{\prime}$ and $f f^{\prime}$ functions. From the computed flux map we compute the equivalent of magnetics measurements. A $1 \%$ noise is added to these measurements. Then in a second step we run the equilibrium reconstruction mode of NICE in $\mathrm{ABB}$ domain that is to say without using the toroidal harmonics step. Functions $\mathcal{A}$ and $\mathcal{B}$ are to be identified in a basis of 11 cubic splines each. The regularization parameters are $\varepsilon_{\mathcal{A}}=\varepsilon_{\mathcal{B}}=10^{-2}$. The computed poloidal flux map is shown on Figure 17. Figure 18 shows the reference and identified $p^{\prime}$ and $f f^{\prime}$ profiles as well as the averaged current density and safety factor. Computed error bars on these profiles are also shown.

ITER vertical displacement event. In this second example an initial ITER plasma is computed thanks to the Ip-fixed static inverse mode of NICE in a first step. It is shown on Figure 19. The desired plasma shape is provided thanks to a parametric representation.

Then in a second step the Ip-fixed quasi-static evolution mode is run. All voltages are set to zero and the time step is $\Delta t=10^{-3} \mathrm{~s}$. At each time step the Newton relative residual converges in few iterations to the tolerance value of $10^{-10}$. A vertical displacement event occurs after approximately $1 \mathrm{~s}$. The evolution of the plasma is shown on Figure 20 and the final poloidal flux map at $t=1.5 \mathrm{~s}$ is shown on Figure 21 .

ITER quasi-static evolution inverse mode. In this third example we use the quasi-static evolution inverse mode of NICE to compute the voltages which drive an ITER limiter plasma upwards. The different desired plasma boundaries as time increases are shown on Figure 22 together with the initial poloidal flux map. The comutation is in Ip-fixed mode and function $\mathcal{A}$ and $\mathcal{B}$ are given by a parametric representation. Each of the 12 voltages time 


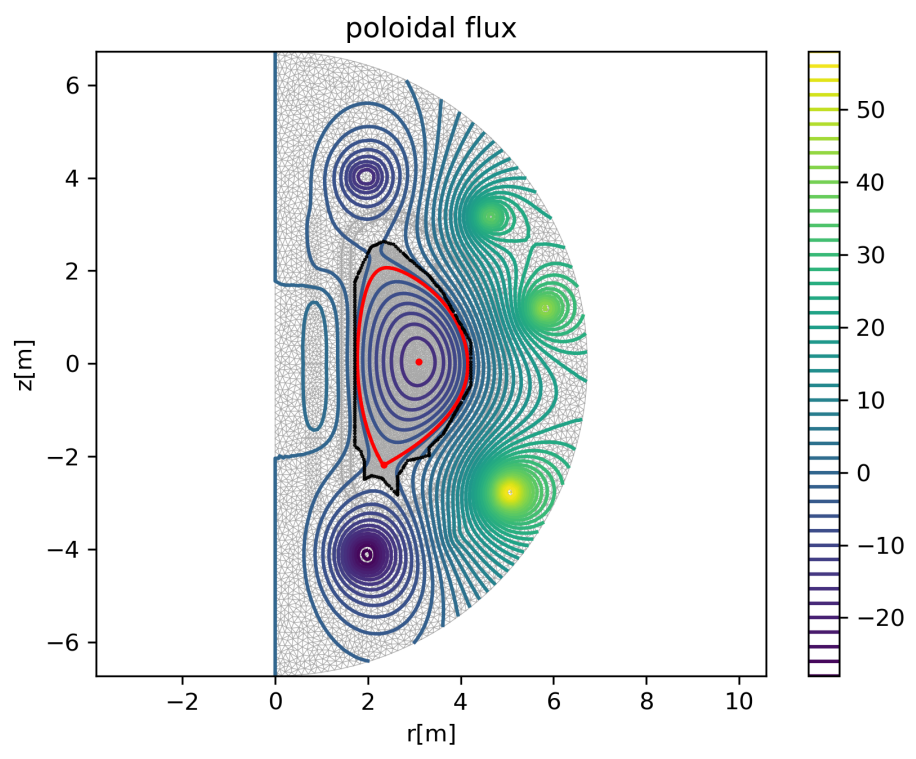

Figure 17: JT60-SA equilibrium reconstruction with synthetic magnetics. Poloidal flux map in ABB domain.

dependent functions to be identified are decomposed in a basis of 11 piecewise linear functions. The weights in the regularization term are chosen as $\sigma_{i}=10^{-5}, 11$ time steps of $0.1 \mathrm{~s}$ are considered. The size of the control vector $\mathbf{u}$ is 132 . The mesh size is 12574 giving a state variable vector $\mathbf{y}$ of size 138314. The QSQP algorithm converges to the tolerance value of $10^{-10}$ in 10 iterations. Figure 23 shows the poloidal flux map at final time with a good match between desired and computed plasma boundaries. Figure 24 shows the voltages computed by the QSQP algorithm.

\section{Conclusion}

This document provides a general overview of the numerical methods implemented in the different effective modes of the code NICE. The code is available on the svn repository of the EUROFUSION gateway. It is still evolving and new features a regularly added. Currently the possibility of using higher order $C^{1}$ finite elements is under development. Other future developments will also deal with the use of the 1D resistive diffusion equation either in a coupling with the quasi-static evolution mode or as an additional 

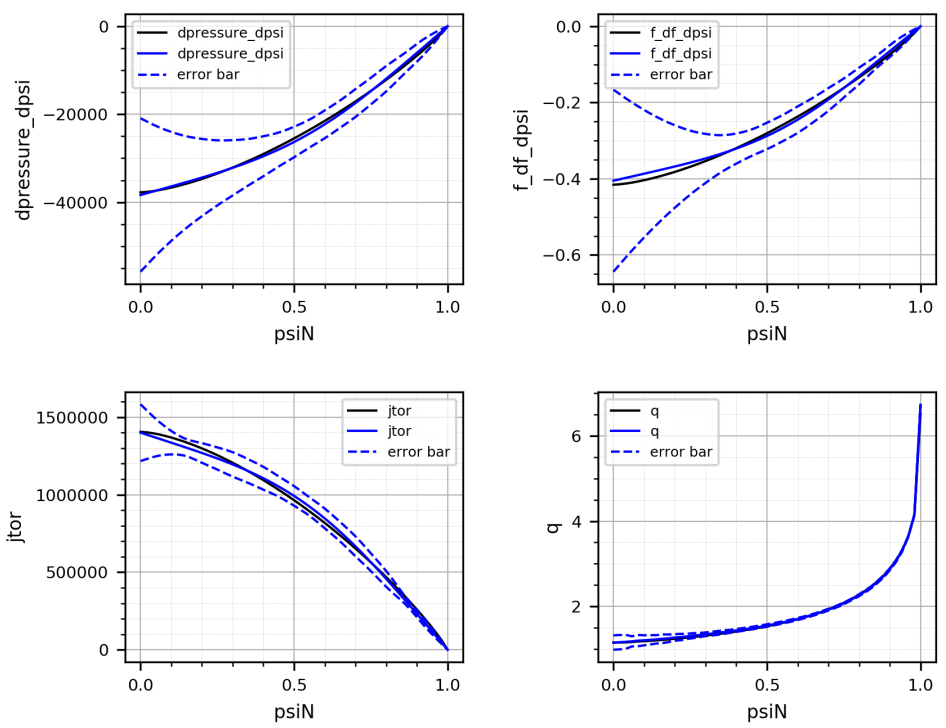

Figure 18: JT60-SA equilibrium reconstruction with synthetic magnetics. Reconstructed $p^{\prime}, f f^{\prime}, j_{\text {tor }}=\left(p^{\prime}+<1 / r^{2}>\left(1 / \mu_{0}\right) f f^{\prime}\right) /<1 / r>$ and safety factor $q$ profiles, and associated computed error bars. The reference equilibrium are in black and the reconstructed ones in blue. 


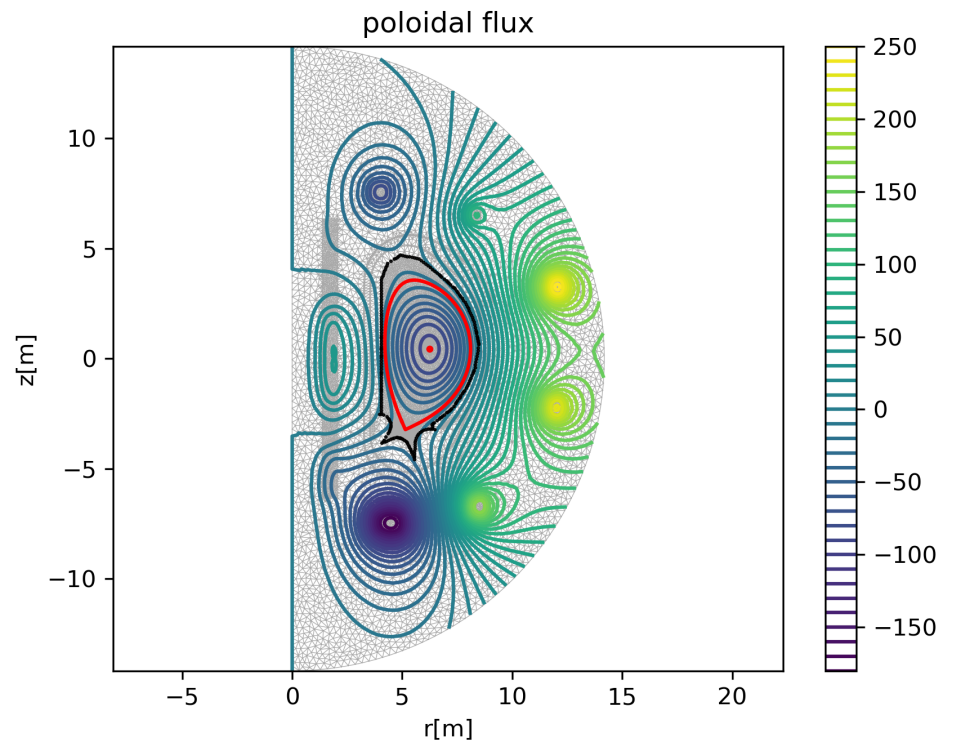

Figure 19: Initial poloidal flux map in ITER geometry.

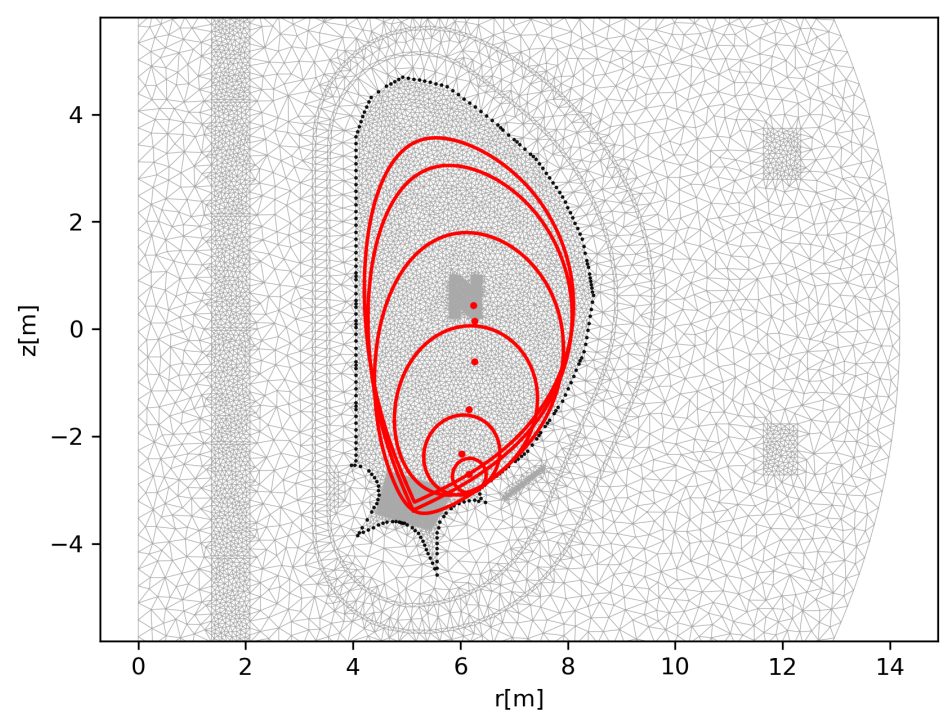

Figure 20: Evolution of the plasma boundary during a VDE in ITER geometry for $t=1$, $1.1,1.2,1.3,1.4$ and $1.5 \mathrm{~s}$ 


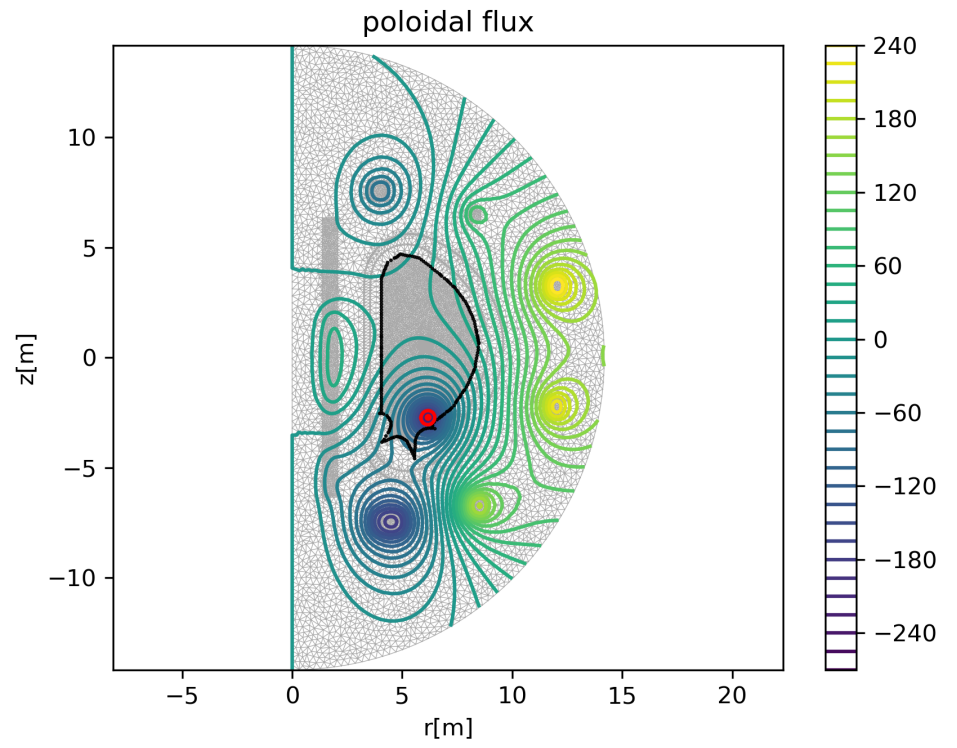

Figure 21: Final poloidal flux map in ITER geometry at $t=1.5 \mathrm{~s}$.

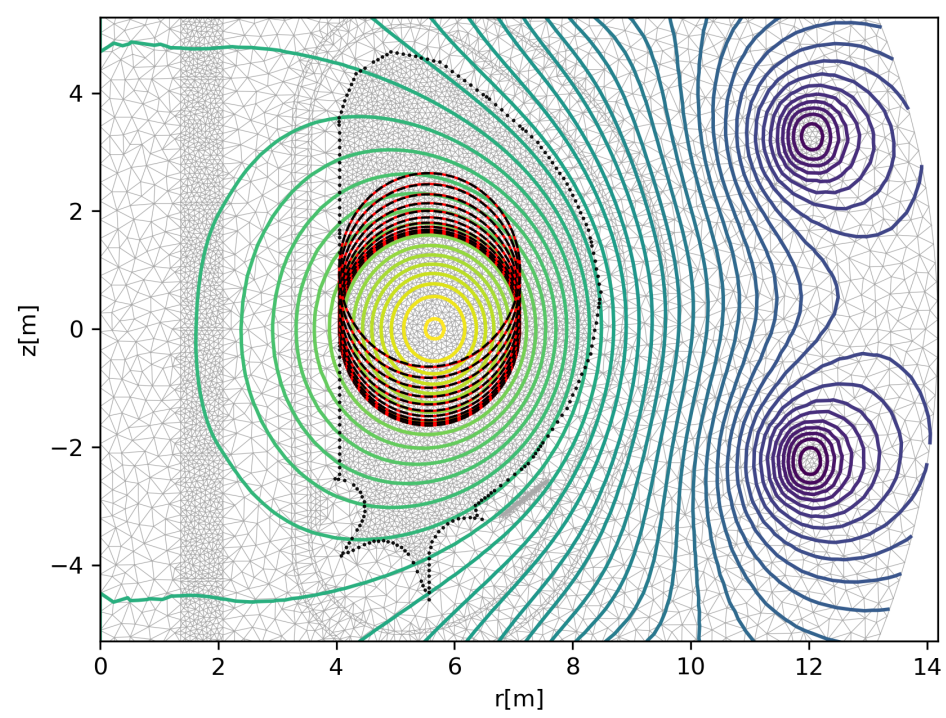

Figure 22: Initial poloidal flux map in ITER geometry and successive desired plasma boundaries for the time evolution. 


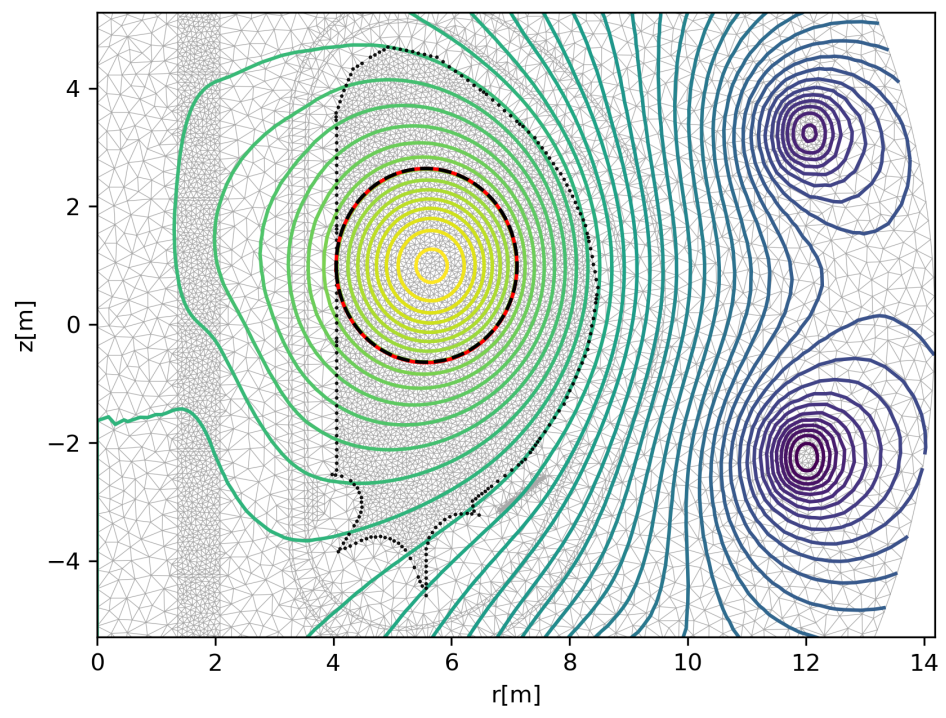

Figure 23: Final poloidal flux map in ITER geometry. Matching computed and desired plasma boundary are shown.

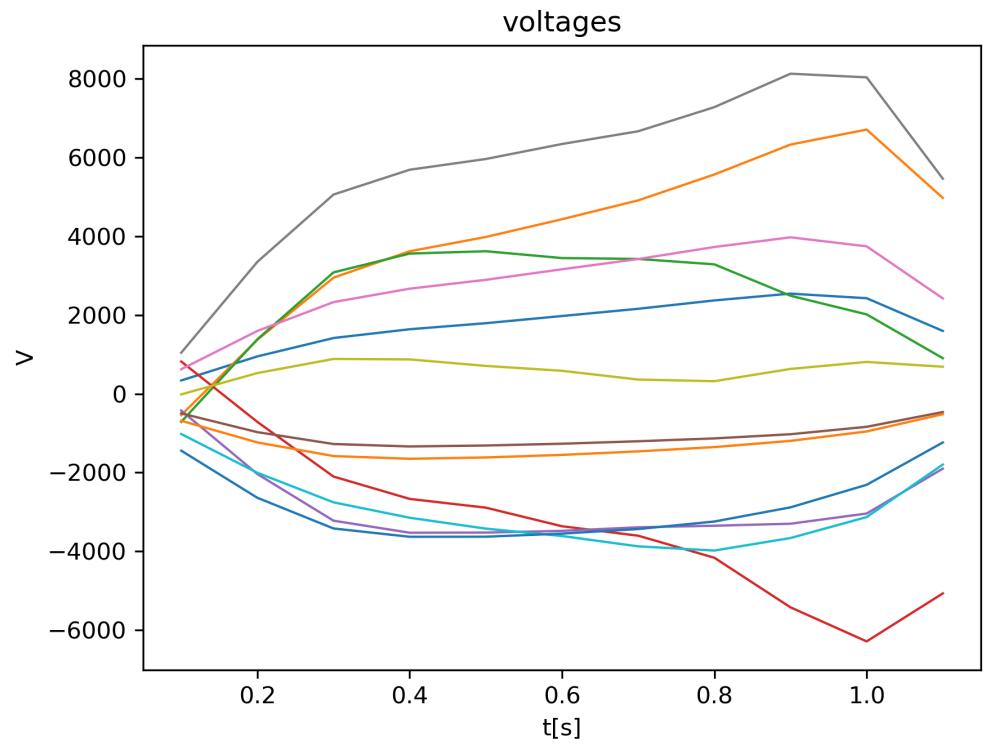

Figure 24: Computed voltages driving the desired plasma boundary evolution. 
constraint in successive equilibrium reconstructions.

\section{Aknowledgments}

The author would like to thank Cédric Boulbe, Hervé Guillard and two anonymous reviewers for their constructive comments on the text, as well as Holger Heumann and Jacques Blum in particular for their inputs on SQP methods.

This work has been carried out within the framework of the EUROfusion Consortium and has received funding from the Euratom research and training programme 2014-2018 and 2019-2020 under grant agreement No 633053. The

views and opinions expressed herein do not necessarily reflect those of the European Commission. 


\section{References}

[1] B. Faugeras, J. Blum, C. Boulbe, P. Moreau, E. Nardon, 2D interpolation and extrapolation of discrete magnetic measurements with toroidal harmonics for equilibrium reconstruction in a Tokamak, Plasma Phys. Control Fusion 56 (2014) 114010.

[2] J. Blum, C. Boulbe, B. Faugeras, Reconstruction of the equilibrium of the plasma in a tokamak and identification of the current density profile in real time, J. Computational Physics 231 (2012) 960-980.

[3] H. Heumann, J. Blum, C. Boulbe, B. Faugeras, G. Selig, J.-M. Ané, S. Brémond, V. Grangirard, P. Hertout, E. Nardon, Quasi-static freeboundary equilibrium of toroidal plasma with CEDRES++: computational methods and applications, J. Plasma Physicsdoi:http://dx. doi.org/10.1017/S0022377814001251.

[4] B. Faugeras, J. Blum, H. Heumann, C. Boulbe, Optimal control of a coupled partial and ordinary differential equations system for the assimilation of polarimetry stokes vector measurements in tokamak free-boundary equilibrium reconstruction with application to ITER, Comput. Phys. Comm. 217 (Supplement C) (2017) 43 - 57. doi:https://doi.org/10.1016/j.cpc.2017.04.003.

URL http://www.sciencedirect.com/science/article/pii/ S0010465517301066

[5] B. Faugeras, F. Orsitto, On the identification of the electron temperature profile from polarimetry Stokes vector measurements in tokamak free-boundary equilibrium reconstruction, Plasma Phys. Control Fusion 61 (11) (2019) 115002. doi:10.1088/1361-6587/ab411a.

[6] J. Blum, H. Heumann, E. Nardon, X. Song, Automating the design of tokamak experiment scenarios, J. Computational Physics 394 (2019) 594-614.

[7] P. Moreau, A. Le-Luyer, P. Spuig, P. Malard, F. Saint-Laurent, J. F. Artaud, J. Morales, B. Faugeras, H. Heumann, B. Cantone, M. Moreau, C. Brun, R. Nouailletas, E. Nardon, B. Santraine, A. Berne, P. Kumari, S. Belsare, The new magnetic diagnostics in the west tokamak, Review of Scientific Instruments 89 (10) (2018) 10J109. arXiv:https://doi. 
org/10.1063/1.5036537, doi:10.1063/1.5036537.

URL https://doi.org/10.1063/1.5036537

[8] F. Imbeaux et al, Design and first applications of the ITER integrated modelling and analysis suite, Nuclear Fusion 5512 (2015) 123006.

[9] S. Pinches et al, Progress in the ITER Integrated Modelling Programme and the Use and Validation of IMAS within the ITER Members, in: 26th IAEA Fusion Energy Conference FEC 2018, Kyoto, Japan, 2016, paper $\mathrm{TH} / \mathrm{P} 2-14$.

[10] R. Coelho, W. Zwingmann, B. Faugeras, E. Giovannozzi, P. McCarthy, E. Suchkov, F. Zaitsev, J. Hollocombe, N. Hawkes, G. Szepesi, L. Appel, S. Silburn, G. Poulipoulis, D. Terranova, Plasma equilibrium reconstruction of JET discharges using the IMAS modelling infrastructure, in: 27th IAEA Fusion Energy Conference IAEA-CN-258 FEC 2018, Gandhinagar, India, 2018.

[11] J. P. Freidberg, Ideal Magnetohydrodynamics, Plenum US, 1987.

[12] J. Blum, Numerical Simulation and Optimal Control in Plasma Physics with Applications to Tokamaks, Series in Modern Applied Mathematics, Wiley Gauthier-Villars, Paris, 1989.

[13] J. Wesson, Tokamaks, The International Series of Monographs in Physics, Oxford University Press, 2004.

[14] J. P. Goedbloed, S. Poedts, Principles of magnetohydrodynamics: with applications to laboratory and astrophysical plasmas, Cambridge university press, 2004.

[15] S. Jardin, Computational methods in plasma physics, Boca Raton, FL : CRC Press/Taylor \& Francis, 2010.

[16] H. Grad, H. Rubin, Hydromagnetic equilibria and force-free fields, Proceedings of the 2nd UN Conf. on the Peaceful Uses of Atomic Energy 31 (1958) 190.

URL http://www-naweb.iaea.org/napc/physics/2ndgenconf/ data/Proceedings1958/papersVol31/Paper25_Vol31.pdf 
[17] V. Shafranov, On magnetohydrodynamical equilibrium configurations, Soviet Journal of Experimental and Theoretical Physics 6 (1958) 545. URL http://adsabs . harvard.edu/abs/1958JETP...6. .545S

[18] R. Lüst, A. Schlüter, Axialsymmetrische magnetohydrodynamische Gleichgewichtskonfigurationen., Z. Naturforsch. A 12 (1957) 850-854.

[19] J. Blum, J. Le Foll, Plasma equilibrium evolution at the resistive diffusion timescale, Computer Physics Reports 1 (7-8) (1984) 465-494. doi:10.1016/0167-7977(84)90013-3.

URL http://www.sciencedirect.com/science/article/pii/ 0167797784900133

[20] R. Albanese, J. Blum, O. Barbieri, On the solution of the magnetic flux equation in an infinite domain, in: EPS. 8th Europhysics Conference on Computing in Plasma Physics (1986), 1986, pp. 41-44.

[21] V. Grandgirard, Modélisation de l'équilibre d'un plasma de tokamak, Ph.D. thesis, Université de Franche-Comté (1999).

[22] B. Faugeras, H. Heumann, FEM-BEM coupling methods for tokamak plasma axisymmetric free-boundary equilibrium computations in unbounded domains, J. Computational Physics 343 (Supplement C) (2017) 201 - 216. doi:https://doi.org/10.1016/j.jcp.2017.04.047.

URL http://www.sciencedirect.com/science/article/pii/ S0021999117303261

[23] J. Nocedal, S. J. Wright, Numerical optimization, 2nd Edition, Springer Series in Operations Research and Financial Engineering, Springer, New York, 2006.

[24] M. Hinze, R. Pinnau, M. Ulbrich, S. Ulbrich, Optimization with PDE constraints, Vol. 23 of Mathematical Modelling: Theory and Applications, Springer, New York, 2009.

[25] M. P.M., F. H., Methods of theoritical physics, Cambridge University press, 1953.

[26] N. Lebedev, Special Functions and their Applications, Dover Publications, 1972. 
[27] M. Abramowitz, S. I.A., Handbook of Mathematical Functions, National bureau of Standards, Washington, DC, 1964.

[28] L. Zakharov, V. Shafranov, Equilibrium of a toroidal plasma with noncircular cross-section, Sov. Phys. Tech. Phys. 18 (2) (1973) 151-156.

[29] B. Braams, The interpretation of tokamak magnetic diagnostics, Plasma Physics and Controlled Fusion 33 (1991) 715.

[30] R. Santos, R. Coelho, P. Rodrigues, B. Faugeras, H. Fernandes, B. B. Carvalho, D. Corona, H. Figueiredo, H. Alves, Plasma boundary reconstruction in ISTTOK using magnetic diagnostic data, Journal of Instrumentation 14 (09) (2019) C09019-C09019. doi:10.1088/1748-0221/ $14 / 09 / \mathrm{c09019}$.

[31] D. O'Brien, J. Ellis, J. Lingertat, Local expansion method for fast plasma boundary identification in JET, Nuclear Fusion 33 (3) (1993) 467-474.

[32] F. Sartori, A. Cenedese, F. Milani, JET real-time object-oriented code for plasma boundary reconstruction, Fusion Eng. Design 66-68 (2003) 735-739.

[33] R. Groebner et al, Progress in quantifying the edge physics of the $\mathrm{H}$ mode regime in DIII-D, Nuclear Fusion 41 (2001) 1789.

[34] B. Faugeras, F. Orsitto, J. Contributors, Equilibrium reconstruction at JET using Stokes model for polarimetry, Nuclear Fusion 58 (10) (2018) 106032. doi:https://doi.org/10.1088/1741-4326/aad751. URL http://stacks .iop. org/0029-5515/58/i=10/a=106032

[35] L. Lao, J. Ferron, R. Geoebner, W. Howl, H. St. John, E. Strait, T. Taylor, Equilibrium analysis of current profiles in Tokamaks, Nuclear Fusion 30 (6) (1990) 1035.

[36] W. Zwingmann, Equilibrium analysis of steady state tokamak discharges, Nuclear Fusion 43 (2003) 842-850.

[37] P. Mc Carthy, P. Martin, W. Schneider, The CLISTE Interpretive Equilibrium Code, Tech. Rep. IPP Report 5/85, Max-Planck-Institut fur Plasmaphysik (1999). 
URL http://physics.ucc.ie/ pjm/people/confs/Cliste_IPP_ 5-85.pdf

[38] J.-M. Moret, B. Duval, H. Le, S. Coda, F. Felici, H. Reimerdes, Tokamak equilibrium reconstruction code LIUQE and its real time implementation, Fusion Eng. Design 91 (0) (2015) 1-15. doi:http://dx.doi.org/10.1016/j.fusengdes.2014.09.019. URL http://www.sciencedirect.com/science/article/pii/ S0920379614005973 